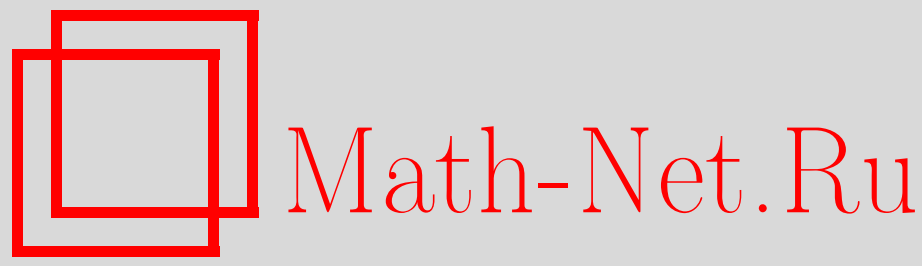

K. С. Платонова, А. В. Боровских, Групповой анализ одномерного уравнения Больцмана III. Условие сохранения физического смысла моментных величин, $T M \Phi$, 2018, том 195, номер 3, 451-482

DOI: https://doi.org/10.4213/tmf9425

Использование Общероссийского математического портала Math-Net.Ru подразумевает, что вы прочитали и согласны с пользовательским соглашением http://www.mathnet.ru/rus/agreement

Параметры загрузки:

IP : 3.93.64.190

26 апреля 2023 г., 10:19:23

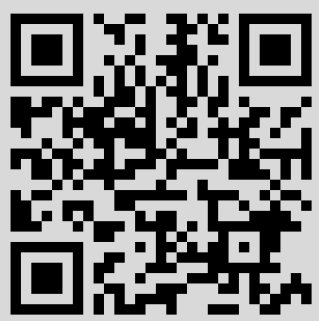




\title{
ГРУППОВОЙ АНАЛИЗ ОДНОМЕРНОГО УРАВНЕНИЯ БОЛЬЦМАНА ІІІ. УСЛОВИЕ СОХРАНЕНИЯ ФИЗИЧЕСКОГО СМЫСЛА МОМЕНТНЫХ ВЕЛИЧИН
}

\begin{abstract}
Проведена групповая классификация одномерного уравнения Больцмана относительно функции $\mathcal{F}=\mathcal{F}(t, x, c)$, характеризующей внешнее силовое поле, в предположении о наличии следующих вытекающих из физических соображений связей между переменными: $d x=c d t, d c=\mathcal{F} d t, d t=0, d x=0$. Показано, что для всех функций $\mathcal{F}$ алгебра конечномерна и ее максимальная размерность равна восьми, что соответствует уравнению с нулевой $\mathcal{F}$.
\end{abstract}

Ключевые слова: уравнение Больцмана, группы симметрий, уравнения газовой динамики, группы эквивалентности.

DOI: https://doi.org/10.4213/tmf9425

\section{1. УРАВНЕНИЕ БОЛЬЦМАНА}

В настоящей работе проводится групповой анализ одномерного уравнения Больщмана [1]

$$
f_{t}+c f_{x}+\mathcal{F} f_{c}=0 \quad(t, x, c \in \mathbb{R}),
$$

описывающего эволюцию распределения частиц жидкости или газа (здесь $t$ - время, $x$ - пространственная координата, $c$ - импульс, $f(t, x, c)$ - фазовая плотность частиц). Функцию $\mathcal{F}(t, x, c)$, отвечающую за силовое поле, мы будем считать заданной (хотя и произвольной).

Следует сказать несколько слов об исходной постановке задачи и связанных с ней проблемах. Собственно говоря, нас интересуют не столько группы симметрий самого́ уравнения Больцмана, сколько их поведение при переходе от уравнения Больцмана к системе уравнений относительно моментных функций

$$
j^{(n)}(t, x)=\int_{-\infty}^{+\infty} f(t, x, c) c^{n} d c .
$$

Работа выполнена при поддержке РФФИ (грант № 15-01-04066).

* Московский государственный университет им. М. В. Ломоносова, Москва, Россия. E-mail: kseniya-plat@yandex.ru 
Конечно, основной перспективный интерес здесь связан с трехмерным уравнением, но, поскольку мы вторгаемся в постановки совершенно новых задач, сначала попытаемся сделать это в случае одномерного уравнения.

Подчеркнем тот факт, что групповой анализ всегда связан с определенной трансформацией задачи, прежде всего со введением произвольных функций. Хорошо известно, что в механике часто используются уравнения, для которых некоторые соотношения могут варьироваться или являться эмпирическими закономерностями. Эти соотношения можно аналитически записать только приближенно, исходя из тех или иных гипотез. Обычно при переходе к математическим постановкам в этом случае используются произвольные функции (на которые могут, конечно, налагаться ограничения типа положительности, монотонности и т. п., но в целом они произвольны). В групповом анализе это является просто правилом, определяемым техникой: он наиболее эффективно работает при минимальном количестве ограничений на класс тех функциональных связей, которые можно варьировать, а неудачные, "лишние", предположения могут резко усложнить анализ. Кроме того, использование произвольных функций иногда приводит к тому, что место механической классификации уравнений занимает математическая - по группам симметрий, как это оказалось в знаменитой работе Овсянникова [2]. Именно по этой причине мы изначально рассматриваем уравнение Больцмана с произвольной функцией $\mathcal{F}(t, x, c)$.

Вторым специфическим свойством группового анализа является существенное (по сравнению с физической постановкой задачи) расширение класса использующихся замен переменных (на физическом языке - замен систем координат). Это резко облегчает вычисления, но порождает проблему интерпретации результатов с точки зрения физического смысла; иногда мы находимся на грани этого смысла, а иногда и за гранью. Поэтому результаты группового анализа приходится постоянно ревизовать на предмет того, насколько при этом не потерян физический смысл, а если потерян, то почему.

Ярким примером подобных задач оказывается как раз групповой анализ уравнения (1), который был проведен в работе [3]. Нетрудно видеть, что уравнение (1) является линейным однородным уравнением в частных производных относительно функции $f$, которое решается стандартным способом, состоящим в нахождении замены переменных $(t, x, c)$, превращающей $(1)$ в простейшее уравнение $f_{t}=0$. Поэтому, на первый взгляд, групповой анализ такого уравнения тривиален: все уравнения эквивалентны между собой, и группы симметрий всех уравнений изоморфны группе симметрий простейшего уравнения, которая легко находится. Но тут обнаруживается одно недоразумение. Допуская произвольные преобразования переменных $(t, x, c)$, мы можем очень просто потерять тот самый физический смысл, потому что величина $c$ - это скорость частицы в пространстве, координаты в котором задаются переменной $x$, и растяжение оси $x$ (при неизменном времени) предполагает также пересчет величины $c$.

Аналогичный, но более яркий пример можно привести для трехмерного случая: как можно повернуть систему координат в пространстве переменных $x$, не повернув ее в пространстве скоростей? Но "в уравнении" это не записано, и преодолеть появившуюся несуразность чисто математическими средствами невозможно. Выход находится в наложении дополнительного условия инвариантности относительно преобразований на дополнительное соотношение $d x=c d t$ (а заодно и на соотношение $d c=\mathcal{F} d t$, с которым оно образует фактически второй закон Ньютона). 
Таким образом, оказывается, что групповой анализ уравнения (1) без условий

$$
d x=c d t, \quad d c=\mathcal{F} d t
$$

(а он дает, как показано в работе [3], совсем другой результат по сравнению с таким же анализом с учетом этих условий) оказывается с физической точки зрения бессмысленным (кстати говоря, видимо, именно этим моментом пренебрегли авторы статьи [4], которые привели левую часть этого уравнения к простейшему виду).

Аналогично "ниоткуда" (с математической точки зрения) появляется еще одно условие, связанное уже не с самим уравнением Больцмана, а с моментными функциями (2). Эти величины имеют физический смысл (функция $j^{(0)}$ интерпретируется как плотность массы, $j^{(1)}-$ как плотность импульса, $j^{(2)}-$ как плотность энергии) в соответствующей среде и вычисляются интегрированием по скоростям соответствующих характеристик частиц, находящихся в заданный момент времени в заданной точке пространства, т. е. формально интегрированием по прямой $t=$ const, $x=$ const. Но при произвольных заменах переменных эта прямая преобразуется в неизвестно какую кривую в пространстве $(t, x, c)$, и интеграл (а с ним и соответствующая величина) теряет физический смысл. Таким образом, для сохранения физического смысла необходимо привнести в постановку задачи условие инвариантности указанных прямых, т. е. условие инвариантности соотношений

$$
d t=0, \quad d x=0 .
$$

Еще один фактор имеет физическое происхождение. Величина

$$
f(t, x, c) d x d c
$$

также имеет физический смысл количества частиц, находящихся в момент времени $t$ в фазовом объеме $d x d c$, и это количество при замене переменных, по идее, должно остаться инвариантным (ведь замена переменных - это только замена системы координат, совокупность частиц как физический объект при этом не меняется). Так возникает еще одно условие.

В результате исходная задача становится нетривиальной. Следует отметить, что некоторые из перечисленных условий упрощают анализ, а некоторые (как, например, последнее) резко усложняют. Поэтому, чтобы довести этот анализ до эффективного результата, нам придется манипулировать условиями: одни из них использовать, а другие на время “забывать”, возвращаясь к ним уже тогда, когда получены какие-то предварительные результаты классификации.

В настоящей работе мы рассматриваем уравнение (1) только в совокупности с условиями (3) и (4), а ситуации, когда к ним добавляется условие инвариантности величины (5), будет посвящена отдельная работа.

\section{2. ГРУППЫ СИММЕТРИЙ И ГРУППЫ ЭКВИВАЛЕНТНОСТИ УРАВНЕНИЙ (1), (3)}

Напомним сначала основные определения и результаты работ [3], [5].

Группой симметрий дифференциального уравнения $Y=0$ называется группа $G$ преобразований, переводящих уравнение $Y=0$ в себя. При этом говорят, что уравнение является инвариантным относительно группы $G$. 
Каждая однопараметрическая подгруппа группы $G$ порождается некоторым векторным полем $\xi$, которое вычисляется как производная по параметру однопараметрической группы при нулевом значении этого параметра. Сама однопараметрическая группа восстанавливается по векторному полю как решение системы обыкновенных дифференциальных уравнений с правой частью, равной $\xi$.

В нашем случае речь идет о преобразовании пространства переменных $(t, x, c, f)$, соответствующие компоненты векторного поля будем обозначать через $(\tau, \xi, \alpha, \eta)$. Однопараметрическая подгруппа восстанавливается по векторному полю как решение системы уравнений

$$
\dot{t}=\tau(t, x, c, f), \quad \dot{x}=\xi(t, x, c, f), \quad \dot{c}=\alpha(t, x, c, f), \quad \dot{f}=\eta(t, x, c, f)
$$

(точка означает дифференцирование по параметру группы). С векторным полем обычно связывают дифференциальный оператор

$$
\Xi=\tau(t, x, c, f) \partial_{t}+\xi(t, x, c, f) \partial_{x}+\alpha(t, x, c, f) \partial_{c}+\eta(t, x, c, f) \partial_{f},
$$

в терминах которого условие инвариантности уравнения $Y=0$ приобретает компактный вид $\left.\Xi Y\right|_{Y=0}=0$ (при этом оператор (7) продолжается стандартным образом на пространство переменных, включающих также производные, в нашем случае производные $f_{t}, f_{x}$ и $f_{c}$ ).

Множество всех операторов, порождающих однопараметрические группы, образует алгебру, т. е. линейное пространство, инвариантное относительно операции коммутации операторов (удовлетворяющей условию Якоби). Если это пространство конечномерно, то по нему можно восстановить связную компоненту группы, содержащую тождественное преобразование, как локально параметризованное множество (многообразие) той же размерности. В настоящей работе мы не строим саму группу, поскольку нам это далее не требуется, а классификацию производим, как это обычно делается, в терминах алгебры Ли операторов (7).

Преобразования, переводящие любое уравнение вида (1) в уравнение того же вида, но с другой функцией $\mathcal{F}$, образуют группу, которая называется группой эквивалентности семейства уравнений вида (1). Группа эквивалентности позволяет разбивать исходное семейство на классы эквивалентных между собой уравнений, редуцируя классификацию всех уравнений к классификации набора представителей соответствующих классов. Эта группа действует уже в пространстве пяти переменных $(t, x, c, f, \mathcal{F})$ и порождается, как и группа симметрий, алгеброй операторов

$$
\widehat{\Sigma}=\tau(t, x, c, f) \partial_{t}+\xi(t, x, c, f) \partial_{x}+\alpha(t, x, c, f) \partial_{c}+\eta(t, x, c, f) \partial_{f}+\Phi(t, x, c, f, \mathcal{F}) \partial_{\mathcal{F}}
$$

В качестве отправной точки приведем два основных результата из работ [3] и [5] о группе симметрий и о группе эквивалентности для уравнения (1), сопровождаемого соотношениями (3).

Обозначим дифференциальный оператор, порождающий уравнение (1), через

$$
\mathcal{D}_{\mathcal{F}}=\partial_{t}+c \partial_{x}+\mathcal{F} \partial_{c}
$$

Теорема 1. Алгебра Ли группь симметрий уравнения (1), сохраняющих дифференциальные соотношения (3), состоит из двух слагаемых: бесконечномерной подалгебры преобразований неизвестной функции

$$
\Xi_{f}=\eta(t, x, c, f) \partial_{f},
$$


где $\eta(t, x, c, f)$ - произвольная функиия, удовлетворяющая условию $\mathcal{D}_{\mathcal{F}} \eta=0, u$ бесконечномерной подалгебры преобразований независимых переменных

$$
\Xi_{t, x, c}=\tau \partial_{t}+(\beta+c \tau) \partial_{x}+\left(\mathcal{D}_{\mathcal{F}} \beta+\mathcal{F} \tau\right) \partial_{c},
$$

где $\tau(t, x, c, f)$ - произвольная функиия, а $\beta(t, x, c)$ удовлетворяет условию

$$
\mathcal{D}_{\mathcal{F}}^{2} \beta-\mathcal{F}_{c} \mathcal{D}_{\mathcal{F}} \beta-\mathcal{F}_{x} \beta=0 .
$$

Теорема 2. Алгебра эквивалентности уравнения (1), сохраняющая дифференииальные соотношения (3), состоит из двух слагаемых: бесконечномерной подалгебры преобразований $\widehat{\Xi}_{f}=\eta(f) \partial_{f}$ функиии $f$ и бесконечномерной подалгебры преобразований независимых переменных $t, x, c$ и $\mathcal{F}$

$$
\widehat{\Xi}_{t, x, c}=-\beta_{c} \partial_{t}+\left(\beta-c \beta_{c}\right) \partial_{x}+\left(\beta_{t}+c \beta_{x}\right) \partial_{c}+\mathcal{D}_{\mathcal{F}}^{2} \beta \partial_{\mathcal{F}},
$$

где $\beta(t, x, c)$ - произвольная функиия.

\section{3. ГРУППЫ СИММЕТРИЙ И ГРУППЫ ЭКВИВАЛЕНТНОСТИ УРАВНЕНИЙ (1), (3), (4)}

Перейдем теперь к изложению основного результата.

Условие инвариантности соотношений (4) и для алгебры симметрий, и для алгебры эквивалентности дает условия $\tau_{c} d c+\tau_{f} d f=\xi_{c} d c+\xi_{f} d f=0$ при любых $d c, d f$, откуда, очевидно, следует, что $\tau=\tau(t, x), \xi=\xi(t, x)$.

Подалгебра алгебры симметрий (10), оставляющая инвариантным условие (4), тем самым приобретает вид $\Xi_{t, x, c}=\tau \partial_{t}+\xi \partial_{x}+\left(\mathcal{D}_{\mathcal{F}}(\xi-c \tau)+\mathcal{F} \tau\right) \partial_{c}$, где $\tau(t, x)$ и $\xi(t, x)$ - произвольные функции, удовлетворяющие уравнению

$$
\begin{aligned}
-\mathcal{F}_{t} \tau & -\mathcal{F}_{x} \xi-\mathcal{F}_{c}\left(\xi_{t}+c \xi_{x}-c \tau_{t}-c^{2} \tau_{x}\right)+\mathcal{F}\left(\xi_{x}-2 \tau_{t}-3 c \tau_{x}\right)+ \\
& +\xi_{t t}+2 c \xi_{t x}+c^{2} \xi_{x x}-c \tau_{t t}-2 c^{2} \tau_{t x}-c^{3} \tau_{x x}=0,
\end{aligned}
$$

которое получается из (11) подстановкой $\beta=\xi-c \tau$.

Что касается алгебры (12), то для нее оказывается, что $\beta_{c c}=0$, и замена $\beta=\xi-c \tau$ приводит к подалгебре

$$
\widehat{\Xi}_{t, x, c}=\tau \partial_{t}+\xi \partial_{x}+\left(\xi_{t}+c\left(\xi_{x}-\tau_{t}\right)-c^{2} \tau_{x}\right) \partial_{c}+\mathcal{D}_{\mathcal{F}}^{2}(\xi-c \tau) \partial_{\mathcal{F}}
$$

с произвольными функциями $\tau=\tau(t, x), \xi=\xi(t, x)$. Как мы видим, это условие достаточно сильно улучшило ситуацию: группа эквивалентности свелась к группе, индуцированной диффеоморфизмами в пространстве $(t, x)$, а группа симметрий индуцирована некоторой подгруппой группы диффеоморфизмов, определяемой уравнением (11). Достаточно большая группа эквивалентности позволяет, как мы увидим ниже, произвести эффективную классификацию уравнений.

Теорема 3. Алгебра симметрий уравнения (1), сохраняющая условия (3), (4), конечномерна для любой функиии $\mathcal{F}(t, x, c)$. Конечномерные нетривиальные алгебры симметрий имеют уравнения с функииями $\mathcal{F}(t, x, c)$, принадлежащими (с точностъю до преобразований из группы эквивалентности (14)) классам, представители которых приведены в табл. 1-3. В каждом случае 1-4, указанном в таблицах, предполагается, что функиия $\mathcal{F}$ не лежит в предыдущем классе. Для остальных функций $\mathcal{F}(t, x, c)$ уравнение (1) не имеет других симметрий, сохраняющих условия (3), (4), кроме тривиальных. 
ТАБлицА 1. Представители классов функций, для которых (1) имеет восьмимерную группу симметрий.

\begin{tabular}{|c||c|ll|}
\hline Случай & $\mathcal{F}(t, x, c)$ & \multicolumn{1}{|c|}{ Базис алгебры симметрий } \\
\hline \hline \multirow{4}{*}{1} & \multirow{2}{*}{$\mathcal{F}=0$} & $\Xi_{1}=\partial_{t}$, & $\Xi_{2}=\partial_{x}$, \\
& & $\Xi_{3}=t \partial_{t}$, & $\Xi_{4}=x \partial_{x}$, \\
& & $\Xi_{5}=t^{2} \partial_{t}+t x \partial_{x}, \quad \Xi_{6}=t x \partial_{t}+x^{2} \partial_{x}$, \\
& & $\Xi_{7}=x \partial_{t}$, & $\Xi_{8}=t \partial_{x}$ \\
\hline
\end{tabular}

ТАБлицА 2. Представители классов функций, для которых (1) имеет трехмерную группу симметрий (здесь $A$ - произвольная константа).

\begin{tabular}{|c|c|c|}
\hline Случай & $\mathcal{F}(t, x, c)$ & Базис алгебры симметрий \\
\hline 2.1 & $\mathcal{F}=A c^{a}$ & $\begin{array}{l}\Xi_{1}=\partial_{t}, \quad \Xi_{2}=\partial_{x} \\
\Xi_{3}=t \partial_{t}+\frac{a-2}{a-1} x \partial_{x}\end{array}$ \\
\hline 2.2 & $\mathcal{F}=A e^{a c}$ & $\begin{array}{c}\Xi_{1}=\partial_{t}, \quad \Xi_{2}=\partial_{x} \\
\Xi_{3}=t \partial_{t}+(x-t / a) \partial_{x}\end{array}$ \\
\hline 2.3 & $\mathcal{F}=A \exp \int \frac{3 c+a}{c^{2}+b c+d} d c$ & $\begin{array}{c}\Xi_{1}=\partial_{t}, \quad \Xi_{2}=\partial_{x}, \\
\Xi_{3}=(x+(a-b) t) \partial_{t}+((a-2 b) x-d t) \partial_{x}\end{array}$ \\
\hline 2.4 & $\mathcal{F}=\frac{A}{x^{3}}$ & $\begin{array}{c}\Xi_{1}=\partial_{t}, \quad \Xi_{2}=t \partial_{t}+\frac{x}{2} \partial_{x} \\
\Xi_{3}=t^{2} \partial_{t}+t x \partial_{x}\end{array}$ \\
\hline 2.5 & $\mathcal{F}=A\left(1+\frac{(t+a c)^{2}}{t^{2}+2 a x}\right)^{3 / 2}$ & $\begin{array}{c}\Xi_{1}=\partial_{t}-\frac{t}{a} \partial_{x}, \quad \Xi_{2}=t \partial_{t}+2 x \partial_{x} \\
\Xi_{3}=-a x \partial_{t}+\left(3 t x+t^{3} / a\right) \partial_{x}\end{array}$ \\
\hline 2.6 & $\mathcal{F}=A\left(\frac{(x-c t)^{2}+c^{2}+1}{x^{2}+t^{2}+1}\right)^{3 / 2}$ & $\begin{array}{c}\Xi_{1}=\left(t^{2}+1\right) \partial_{t}+t x \partial_{x} \\
\Xi_{2}=t x \partial_{t}+\left(x^{2}+1\right) \partial_{x} \\
\Xi_{3}=-x \partial_{t}+t \partial_{x}\end{array}$ \\
\hline
\end{tabular}

ТАБлицА 3. Представители классов функций, для которых (1) имеет двухи одномерную группы симметрий (здесь $\mathcal{F}, T$ - произвольные функции).

\begin{tabular}{|c||c|c|}
\hline Случай & $\mathcal{F}(t, x, c)$ & Базис алгебры симметрий \\
\hline \hline 3.1 & $\mathcal{F}=\mathcal{F}(c)$ & $\Xi_{1}=\partial_{t}, \quad \Xi_{2}=\partial_{x}$ \\
\hline \multirow{2}{*}{3.2} & $\mathcal{F}=\frac{T(c)}{t}$ & $\Xi_{1}=\partial_{x}, \quad \Xi_{2}=t \partial_{t}+x \partial_{x}$ \\
\hline \multirow{2}{*}{4} & $\mathcal{F}=\mathcal{F}(t, c)$ & $\Xi_{1}=\partial_{x}$ \\
& $\mathcal{F}=\mathcal{F}(x, c)$ & $\Xi_{1}=\partial_{t}$ \\
\hline
\end{tabular}




\section{4. ДОКАЗАТЕЛЬСТВО ТЕОРЕМЫ}

Непосредственной подстановкой в выражение (13) проверяется, что всем перечисленным в формулировке теоремы функциям $\mathcal{F}(t, x, c)$ соответствуют указанные алгебры симметрий. Поэтому нам нужно фактически показать, что уравнения (1) с соответствующими функциями не имеют более широких алгебр симметрий, чем приведенные в формулировке теоремы.

Сначала мы покажем, что выполнено первое утверждение теоремы: алгебра симметрий уравнения (1), сохраняющая (3) и (4), не может быть бесконечномерной (даже более того, иметь размерность более восьми) ни для какой функции $\mathcal{F}(t, x, c)$.

Далее мы воспользуемся классическим результатом Ли-Олвера - классификацией алгебр Ли групп преобразований двумерного пространства. Этот результат был получен Ли в [6] (для комплексного пространства), а потом, в вещественном случае, уточнен в [7]. На самом деле нам понадобится только простое следствие из этого результата: все эти алгебры Ли, кроме одной, имеют двумерные подалгебры. Исключение составляет алгебра со структурой $\mathfrak{s o}(3)$, которую рассмотрим отдельно.

Далее мы сосредоточим основное внимание на уравнениях, допускающих двумерные группы симметрий, из которых выделяются отдельные семейства, приведенные в табл. 1,2 .

4.1. Конечномерность алгебры симметрий. Предположим, что уравнение (1) с некоторой функцией $\mathcal{F}(t, x, c)$ имеет алгебру симметрий размерности не менее семи. В этом случае мы можем написать как минимум семь уравнений вида (13) для каждого оператора $\Xi^{i}=\tau^{i} \partial_{t}+\xi^{i} \partial_{x}, i=1, \ldots, 7$. Полученную систему уравнений можно рассматривать как линейную однородную алгебраическую систему относительно $\mathcal{F}_{t}, \mathcal{F}_{x}, \mathcal{F}_{c}, c \mathcal{F}_{c}, c^{2} \mathcal{F}_{c}-3 c \mathcal{F}, \mathcal{F}$ и единицы. Поскольку эта система имеет нетривиальное решение, ее определитель равен нулю:

$$
\left|\begin{array}{ccccccc}
\tau^{1} & \xi^{1} & \xi_{t}^{1} & \xi_{x}^{1}-\tau_{t}^{1} & -\tau_{x}^{1} & \xi_{x}^{1} & -\left(\xi_{t t}^{1}+c\left(2 \xi_{t x}^{1}-\tau_{t t}^{1}\right)+c^{2}\left(\xi_{x x}^{1}-2 \tau_{t x}^{1}\right)-c^{3} \tau_{x x}^{1}\right) \\
\vdots & \vdots & \vdots & \vdots & \vdots & \vdots & \vdots \\
\tau^{7} & \xi^{7} & \xi_{t}^{7} & \xi_{x}^{7}-\tau_{t}^{7} & -\tau_{x}^{7} & \xi_{x}^{7} & -\left(\xi_{t t}^{7}+c\left(2 \xi_{t x}^{7}-\tau_{t t}^{7}\right)+c^{2}\left(\xi_{x x}^{7}-2 \tau_{t x}^{7}\right)-c^{3} \tau_{x x}^{7}\right)
\end{array}\right|=0 .
$$

В силу независимости $\tau^{i}$ и $\xi^{i}$ от $c$ мы получаем из этого условия четыре разных определителя, каждый из которых равен нулю. Условие линейной зависимости столбцов каждого из этих определителей переписывается в виде дифференциального уравнения, которому должны удовлетворять все пары $\left(\tau^{i}, \xi^{i}\right)$ (причем, поскольку нетривиальность решения указанной системы гарантированно содержится в последней компоненте, можно утверждать, что последний столбец выражается через остальные). Получим

$$
\xi_{t t}=L_{11}^{1}(\tau, \xi), \quad 2 \xi_{t x}-\tau_{t t}=L_{11}^{2}(\tau, \xi), \quad \xi_{x x}-2 \tau_{t x}=L_{11}^{3}(\tau, \xi), \quad \tau_{x x}=L_{11}^{4}(\tau, \xi),
$$

где через $L_{i j}^{k}(\tau, \xi)$ обозначены линейные дифференциальные операторы (с, вообе говоря, переменными коэффициентами), имеющие порядок $i$ относительно производных функции $\tau$ и порядок $j$ относительно производных функции $\xi$, индекс $k$ нумерует эти дифференциальные операторы.

Полученная система дифференциальных уравнений имеет только конечномерное пространство решений. В этом легко убедиться: воспользовавшись условиями согласования производных функции $\xi$ в первых трех уравнениях, мы получаем два 
соотношения

$$
\tau_{t t t}=L_{21}^{5}(\tau, \xi), \quad \tau_{t t x}=L_{21}^{6}(\tau, \xi) .
$$

Добавление к ним соотношений

$$
\tau_{t x x}=L_{21}^{7}(\tau, \xi), \quad \tau_{x x x}=L_{21}^{8}(\tau, \xi),
$$

которые получаются дифференцированием последнего уравнения в (15) и первых трех уравнений из (15), дает нам линейную систему относительно функций $\tau$ и $\xi$ в нормальной форме, размерность пространства решений которой не превосходит количества начальных условий для функций $\tau, \xi$ и их производных (для $\tau$ производных до второго порядка, а для $\xi$ до первого порядка). Общее количество таких начальных условий равно девяти, но на самом деле восьми, поскольку в силу последнего из уравнений (15) начальное значение для $\tau_{x x}$ определяется по остальным однозначно. Значит, интересующая нас алгебра симметрий не может иметь размерность больше восьми.

Как уже отмечалось выше, все конечные группы преобразований плоскости были классифицированы в [6]. Согласно уточнению этой классификации [7] все конечные действительные алгебры Ли групп преобразований плоскости $\mathbb{R}^{2}$ либо одномерны, либо имеют двумерную подалгебру, либо эквивалентны алгебре вращений $\mathfrak{s o}(3, \mathbb{R})$. Функции $\mathcal{F}(t, x, c)$, которым соответствуют двумерные и одномерные алгебры, мы рассмотрим далее, а в следующем пункте найдем, каким уравнениям вида (1) соответствует алгебра со структурой $\mathfrak{s o}(3, \mathbb{R})$.

4.2. Уравнение, допускающее алгебру симметрий $\mathfrak{s o}(3, \mathbb{R})$. Нам удобно использовать следующую реализацию алгебры $\mathfrak{s o}(3)$ :

$$
\Xi_{1}=-x \partial_{t}+t \partial_{x}, \quad \Xi_{2}=t x \partial_{t}+\left(x^{2}+1\right) \partial_{x}, \quad \Xi_{3}=\left(t^{2}+1\right) \partial_{t}+t x \partial_{x} ;
$$

эта алгебра имеет то преимущество, что для нее уравнение (13) оказывается однородным.

Проверим, какие функции $\mathcal{F}(t, x, c)$ обладают данной алгеброй. Подставим операторы $\Xi_{1}, \Xi_{2}$ и $\Xi_{3}$ в (13), получим систему уравнений

$$
\begin{aligned}
& x \mathcal{F}_{t}-t \mathcal{F}_{x}-\mathcal{F}_{c}\left(1+c^{2}\right)+3 c \mathcal{F}=0, \\
& t x \mathcal{F}_{t}+\left(1+x^{2}\right) \mathcal{F}_{x}+c \mathcal{F}_{c}(x-t c)+3 t c \mathcal{F}=0, \\
& \left(1+t^{2}\right) \mathcal{F}_{t}+t x \mathcal{F}_{x}+\mathcal{F}_{c}(x-t c)+3 t \mathcal{F}=0 .
\end{aligned}
$$

Линейным комбинированием эта система приводится к виду

$$
\begin{aligned}
& x \mathcal{F}_{t}-t \mathcal{F}_{x}-\mathcal{F}_{c}\left(1+c^{2}\right)+3 c \mathcal{F}=0, \\
& \left(1+t^{2}+x^{2}\right) \mathcal{F}_{x}+\mathcal{F}_{c}(t+c x)=0, \\
& \left(1+t^{2}+x^{2}\right) \mathcal{F}_{t}+c\left(1+t^{2}+x^{2}\right) \mathcal{F}_{x}+3 \mathcal{F}(t+c x)=0 .
\end{aligned}
$$

Из последнего уравнения получаем, что $\mathcal{F}=\Phi(x-c t, c)\left(1+x^{2}+t^{2}\right)^{-3 / 2}$, а подстановка этого выражения в первое уравнение дает соотношение

$$
a c \Phi_{a}+\left(1+c^{2}\right) \Phi_{c}-3 c \Phi=0
$$


где $a$ обозначает первый аргумент функции $\Phi$. Отсюда мы получаем

$$
\Phi(a, c)=\left(1+c^{2}\right)^{3 / 2} \Psi\left(\frac{a^{2}}{1+c^{2}}\right) \quad \Longrightarrow \quad \mathcal{F}=\frac{\left(1+c^{2}\right)^{3 / 2} \Psi\left(\frac{(x-c t)^{2}}{1+c^{2}}\right)}{\left(1+x^{2}+t^{2}\right)^{3 / 2}}
$$

Наконец, подстановка полученного выражения во второе уравнение системы приводит уже к обыкновенному дифференциальному уравнению $2 \Psi^{\prime}(b)(1+b)-3 \Psi=0$, откуда, очевидно, имеем $\Psi=N(1+b)^{3 / 2}$, что и дает функцию

$$
\mathcal{F}=N \frac{\left((x-c t)^{2}+1+c^{2}\right)^{3 / 2}}{\left(1+x^{2}+t^{2}\right)^{3 / 2}},
$$

указанную в формулировке теоремы.

Обратно, подстановка функции $\mathcal{F}$ указанного вида в выражение $(13)$ в силу иррациональной зависимости его от $c$ немедленно влечет равенство нулю свободного члена этого уравнения, т. е.

$$
\xi_{t t}=2 \xi_{t x}-\tau_{t t}=\xi_{x x}-2 \tau_{t x}=\tau_{x x}=0,
$$

откуда $\tau=A t^{2}+B t x+\cdots, \xi=A t x+B x^{2}+\cdots$, где многоточиями обозначены линейные функции. Подстановка функций $\tau$ и $\xi$ в (13) приводит к указанной алгебре. Впрочем, можно заметить, что последних рассуждений можно было бы и не проводить, поскольку из результатов работ [6], [7] следует, что единственной конечномерной алгеброй преобразований плоскости $\mathbb{R}^{2}$, содержащей $\mathfrak{s o}(3)$, является только сама эта алгебра $\mathfrak{s o}(3)$.

\section{3. Уравнения с двумерными коммутативными алгебрами симметрий.} Как уже отмечалось выше, любая конечномерная алгебра симметрий, кроме $\mathfrak{s o}(3)$, содержит двумерную подалгебру. Поэтому ниже мы найдем все уравнения, допускающие двумерную алгебру симметрий, и уже среди них будем искать уравнения с алгебрами бо́льших размерностей.

Любую двумерную алгебру $\Xi=A \Xi_{1}+B \Xi_{2}$ за счет выбора подходящего базиса можно привести к одному из двух канонических случаев: $\left[\Xi_{1}, \Xi_{2}\right]=0$ (коммутативная алгебра) и $\left[\Xi_{1}, \Xi_{2}\right]=\Xi_{2}$ (некоммутативная алгебра); мы далее рассмотрим каждый их этих двух случаев.

Пусть сначала алгебра коммутативна. Тогда без ограничения общности можно считать, что она имеет вид $\Xi=A \partial_{x}+B \partial_{t}$. Действительно, один из операторов заменой переменных из группы эквивалентности можно привести к виду $\Xi_{1}=\partial_{x}$, при этом соответствующая замена $\bar{t}=\varphi(t, x), \bar{x}=\psi(t, x)$ находится как решение системы уравнений $\Xi_{1} \varphi=0, \Xi_{2} \psi=1$. Тогда из условий коммутирования для оператора $\Xi_{2}=\tau_{2} \partial_{t}+\xi_{2} \partial_{x}$ получаем $\tau_{2}=\tau_{2}(t), \xi_{2}=\xi_{2}(t)$. В этом случае мы можем найти замену переменных из группы эквивалентности, которая переведет $\left(\tau_{2}, \xi_{2}\right)$ в $(1,0)$ и сохранит первое векторное поле:

$$
\phi(t)=\int \frac{1}{\tau_{2}(t)} d t, \quad \psi(t, x)=x-\int \frac{\xi_{2}(t)}{\tau_{2}(t)} d t .
$$

Таким образом, можно сразу считать, что речь идет об алгебре с базисом $\partial_{t}, \partial_{x}$. Подставляя компоненты базиса в уравнение $(13)$, получаем, что $\mathcal{F}=\mathcal{F}(c)$. Очевидно, что для любой такой функции $\mathcal{F}$ соответствующая группа симметрий будет содержать как минимум выбранную нами двумерную алгебру. Найдем среди этих функций те, для которых алгебра будет иметь размерность больше двух. 
4.4. Большие алгебры симметрий, содержащие двумерную коммутативную алгебру. Подставим функцию $\mathcal{F}(c)$ в уравнение $(13)$ и упростим его:

$$
\begin{aligned}
-\mathcal{F}_{c}\left(\xi_{t}+c\left(\xi_{x}-\tau_{t}\right)-c^{2} \tau_{x}\right) & +\mathcal{F}\left(\xi_{x}-2 \tau_{t}-3 c \tau_{x}\right)+ \\
& +\xi_{t t}+c\left(2 \xi_{t x}-\tau_{t t}\right)+c^{2}\left(\xi_{x x}-2 \tau_{t x}\right)-c^{3} \tau_{x x}=0 .
\end{aligned}
$$

Дальнейшие рассуждения состоят в том, что, используя факт независимости функции $\mathcal{F}$ от $t$ и $x$, мы можем зафиксировать в (17) некоторые значения этих переменных, решить получившееся обыкновенное дифференциальное уравнение, получить анзац для функции $\mathcal{F}$, а затем подставить ее обратно в уравнение $(17)$, получив систему дифференциальных уравнений относительно $\tau$ и $\xi$. В результате возникает довольно большое количество ветвлений вариантов, приводящих во многих случаях к одним и тем же функциям. Поэтому мы для сокращения рассуждений сначала рассмотрим некоторые заранее выделенные классы функций, а затем покажем, что для функций, в этих классах не лежащих, алгебра симметрий не может иметь размерность больше двух.

Отметим несколько полезных в дальнейшем замен переменных, лежащих в группе эквивалентности уравнений (1), (3), (4), которые позволяют “упрощать" функцию $\mathcal{F}(t, x, c)$ (под словами "замена переменных переводит $\mathcal{F}_{1}$ в $\mathcal{F}_{2}$ " будет всегда пониматься, что замена переводит уравнение $(1)$ с функцией $\mathcal{F}_{1}$ в уравнение $(1)$ с функцией $\left.\mathcal{F}_{2}\right)$ :

1) замена $\bar{t}=t, \bar{x}=x+a t, \bar{c}=c+a$ переводит $\mathcal{F}(t, x, c)$ в $\overline{\mathcal{F}}(\bar{t}, \bar{x}-a \bar{t}, \bar{c}-a)$;

2) замена $\bar{t}=t, \bar{x}=x-a t^{2} / 2, \bar{c}=c-a t$ переводит $\mathcal{F}=a$ в $\overline{\mathcal{F}}=0$;

3) замена $\bar{t}=x, \bar{x}=t, \bar{c}=1 / c$ переводит $\mathcal{F}(t, x, c)$ в $\overline{\mathcal{F}}(\bar{t}, \bar{x}, \bar{c})=-\bar{c}^{3} \mathcal{F}(\bar{x}, \bar{t}, 1 / \bar{c})$;

4) замена $\bar{t}=x, \bar{x}=e^{a t}, \bar{c}=a e^{a t} / c$ переводит $\mathcal{F}(c)=a c+b c^{2}$ в $\overline{\mathcal{F}}(\bar{c})=-b \bar{c}$;

5) замена $\bar{t}=\sin (\mu t) e^{D x}, \bar{x}=\cos (\mu t) e^{D x}, \bar{c}=\frac{D \cos (\mu t) c-\mu \sin (\mu t)}{D \sin (\mu t) c+\mu \cos (\mu t)}$, где $\mu=\sqrt{D G}$, переводит $\mathcal{F}=D c^{2}+G$ с $D G>0$ в $\overline{\mathcal{F}}=0$.

Итак, рассмотрим отдельно алгебры симметрий, соответствующие уравнению (1) с функцией $\mathcal{F}(c)$, принадлежащей какому-либо из шести классов, упомянутых ниже в леммах 1-6. Напомним, что все дальнейшие результаты формулируются с точностью до преобразований из группы эквивалентности.

Лемма 1. Из функиий вида $\mathcal{F}(c)=Q c^{5}+A c^{4}+B c^{3}+D c^{2}+E c+G$ алгебру симметрий размерности больше двух имеют следующие:

- $\mathcal{F}(c)=B c^{3}+D c^{2}+E c+G$, уравнения с любыми такими функииями $\mathcal{F}$ эквивалентны уравнению с $\mathcal{F} \equiv 0$, алгебра которого восвмимерна (см. табл. 1);

- $\mathcal{F}(c)=Q c^{5}$, при этом алгебра трехмерна: $\Xi_{1}=\partial_{t}, \Xi_{2}=\partial_{x}, \Xi_{3}=t \partial_{t}+(3 / 4) x \partial_{x}$;

- $\mathcal{F}(c)=A c^{4}$, при этом алгебра трехмерна: $\Xi_{1}=\partial_{t}, \Xi_{2}=\partial_{x}, \Xi_{3}=t \partial_{t}+(2 / 3) x \partial_{x}$.

ДокАзАТЕЛЬСтво. Подставим функцию $\mathcal{F}$ заданного вида в уравнение $(17)$ :

$$
\begin{aligned}
& -\left(5 Q c^{4}+4 A c^{3}+3 B c^{2}+2 D c+E\right)\left(\xi_{t}+c\left(\xi_{x}-\tau_{t}\right)-c^{2} \tau_{x}\right)+\xi_{t t}+c\left(2 \xi_{t x}-\tau_{t t}\right)+ \\
& \quad+c^{2}\left(\xi_{x x}-2 \tau_{t x}\right)-c^{3} \tau_{x x}+\left(Q c^{5}+A c^{4}+B c^{3}+D c^{2}+E c+G\right)\left(\xi_{x}-2 \tau_{t}-3 c \tau_{x}\right)=0 .
\end{aligned}
$$


Мы получили многочлен по $c$, тождественно равный нулю, следовательно, все его коэффициенты при степенях $c$ равны нулю:

$$
\begin{gathered}
2 Q \tau_{x}=0, \quad 4 Q \xi_{x}-3 Q \tau_{t}-A \tau_{x}=0, \quad 5 Q \xi_{t}+3 A \xi_{x}-2 A \tau_{t}=0, \\
4 A \xi_{t}-B \tau_{t}+2 B \xi_{x}+D \tau_{x}+\tau_{x x}=0, \quad 3 B \xi_{t}+D \xi_{x}+2 E \tau_{x}-\xi_{x x}+2 \tau_{t x}=0, \\
2 D \xi_{t}+E \tau_{t}+3 G \tau_{x}-2 \xi_{t x}+\tau_{t t}=0, \quad E \xi_{t}-G \xi_{x}+2 G \tau_{t}-\xi_{t t}=0 .
\end{gathered}
$$

1. Если $Q \neq 0$, то $\mathcal{F}$ - многочлен пятой степени, у которого всегда есть вещественный корень, обозначим его как $c=M$. Сделаем замену переменных вида $\bar{t}=t, \bar{x}=x-M t, \bar{c}=c-M$. Эта замена переводит функцию $\mathcal{F}$ в функцию вида $\mathcal{F}=Q c^{5}+A c^{4}+B c^{3}+D c^{2}+E c$ (с новыми коэффициентами). Система (18) при этом приобретает вид

$$
\begin{gathered}
\tau_{x}=0, \quad 4 Q \xi_{x}-3 Q \tau_{t}-A \tau_{x}=0, \quad 5 Q \xi_{t}+3 A \xi_{x}-2 A \tau_{t}=0, \\
4 A \xi_{t}-B \tau_{t}+2 B \xi_{x}+D \tau_{x}+\tau_{x x}=0, \quad 3 B \xi_{t}+D \xi_{x}+2 E \tau_{x}-\xi_{x x}+2 \tau_{t x}=0, \\
2 D \xi_{t}+E \tau_{t}-2 \xi_{t x}+\tau_{t t}=0, \quad E \xi_{t}-\xi_{t t}=0 .
\end{gathered}
$$

Из первого соотношения имеем $\tau=\tau(t)$, тогда

$$
\xi=\frac{3}{4} \tau_{t} x+n, \quad 15 Q \tau_{t t} x+20 Q n_{t}+A \tau_{t}=0 \quad \Longrightarrow \quad \tau_{t t}=0, \quad n=-\frac{A k t}{20 Q}+n_{2} .
$$

Остаются четыре уравнения: $k\left(5 Q B-2 A^{2}\right)=0, k(5 Q D-A B)=0, k(10 Q E-A D)=0$ и $A E k=0$.

Случай $k=0$ приводит к двумерной алгебре. Если $k \neq 0$, то

$$
B=\frac{2 A^{2}}{5 Q}, \quad D=\frac{2 A^{3}}{25 Q^{2}}, \quad E=\frac{2 A^{4}}{250 Q^{3}} ;
$$

из уравнения $A E k=0$ имеем $A=0$, т. е. $\tau=k t+m, \xi=3 k x / 4+n_{2}$ есть трехмерная алгебра для $\mathcal{F}=Q c^{5}$.

2. Пусть $Q=0, A \neq 0$. Тогда из (18) получаем уравнение $\tau_{x}=0$ и систему

$$
\begin{gathered}
A\left(3 \xi_{x}-2 \tau_{t}\right)=0, \quad 4 A \xi_{t}-B \tau_{t}+2 B \xi_{x}=0, \quad 3 B \xi_{t}+D \xi_{x}-\xi_{x x}=0, \\
2 D \xi_{t}+E \tau_{t}-2 \xi_{t x}+\tau_{t t}=0, \quad E \xi_{t}-G \xi_{x}+2 G \tau_{t}-\xi_{t t}=0 .
\end{gathered}
$$

Из первого уравнения этой системы следует, что $\xi=2 \tau_{t} x / 3+n$. Подставим это в следующее уравнение, имеем $8 A \tau_{t t} x+12 A n_{t}+B \tau_{t}=0$, отсюда $\tau=k t+m$ и $n=-B k t / 12 A+n_{2}$. Остальные уравнения сводятся к алгебраическим уравнениям $k\left(8 A D-3 B^{2}\right)=k(6 A E-B D)=0, k(16 A G-B E)=0$.

Если $k=0$, то алгебра двумерная. Если $k \neq 0$, то

$$
D=\frac{3 B^{2}}{8 A}, \quad E=\frac{B^{3}}{16 A^{2}}, \quad G=\frac{B^{4}}{256 A^{3}} .
$$

Таким образом, для функции $\mathcal{F}=A(c+B / 4 A)^{4}$ мы получаем, что $\tau=k t+m$, $\xi=k(2 x / 3-B t / 12 A)+n_{2}$ есть трехмерная алгебра. Указанная функция заменой переменных вида 1 приводится к виду $\mathcal{F}=A c^{4}$, для которой трехмерная алгебра симметрий есть $\tau=k t+m, \xi=2 k x / 3+n_{2}$. 
3. Теперь рассмотрим случай $Q=A=0, B \neq 0$. Заменой вида 1 приведем многочлен к случаю $G=0$. С помощью замены переменных вида 3 приведем функцию $\mathcal{F}=B c^{3}+D c^{2}+E c$ к виду $\mathcal{F}=B+D c+E c^{2}$, т. е. к случаям 4-6, рассматриваемым ниже.

4. Пусть $Q=A=B=0, D \neq 0$. Заменой переменных $\bar{t}=t, \bar{x}=x+E t / 2 D$, $\bar{c}=c+E / 2 D$ приведем функцию $\mathcal{F}$ к виду $\mathcal{F}=D c^{2}+G$. Далее имеем следующее:

a) пусть $D G<0$, тогда с помощью сдвига переменной $c$ мы можем привести функцию $\mathcal{F}$ к виду $\mathcal{F}=E c+D c^{2}$; воспользуемся заменой вида 4 и получим $\mathcal{F}=-D c$; далее повторно применим замену 4 и получим $\mathcal{F}=0$;

б) пусть $G=0$, этот случай заменами вида 3 и 4 приводится к случаю $\mathcal{F}=0$;

в) пусть $D G>0$, здесь приведение к случаю $\mathcal{F}=0$ осуществляется с помощью замены 5 .

5. Пусть $Q=A=B=D=0, E \neq 0$. С учетом замены переменных вида 1 можно считать, что $\mathcal{F}=E c$. Заменой переменных вида 4 функция данного вида приводится к $\mathcal{F}=0$.

6. Пусть $Q=A=B=D=E=0$, тогда $\mathcal{F}=G$, и заменой переменных вида 2 эту функцию можно привести к $\mathcal{F}=0$.

7. Пусть $\mathcal{F} \equiv 0$. Система (18) в этом случае упрощается до $\tau_{x x}=0, \xi_{x x}=2 \tau_{t x}$, $2 \xi_{t x}=\tau_{t t}, \xi_{t t}=0$. Решение этой системы имеет вид $\tau=k_{1} t x+k_{2} x+m_{1} t^{2}+m_{2} t+m_{3}$, $\xi=k_{1} x^{2}+m_{1} t x+n_{1} x+l_{1} t+l_{2}$ и определяет алгебру, приведенную в табл. 1.

Лемма доказана.

Лемма 2. Для функиий вида

$$
\mathcal{F}(c)=A c^{4}+B c^{3}+D c^{2}+E c+G+\frac{K}{c+M}, \quad K \neq 0,
$$

алгебра симметрий двумерна (за исключением случаев, эквивалентных перечисленным в лемме 1).

Доказательство. Сделаем замену переменных вида $\bar{t}=t, \bar{x}=x+M t, \bar{c}=c+M$. Эта замена принадлежит группе эквивалентности и переводит функцию $\mathcal{F}$ вида (19) в функцию вида

$$
\mathcal{F}(c)=A c^{4}+B c^{3}+D c^{2}+E c+G+\frac{K}{c} .
$$

Подставим эту функцию в уравнение (17) и умножим на $c^{2}$, получим

$$
\begin{aligned}
& -\left(4 A c^{3}+3 B c^{2}+2 D c+E\right)\left(\xi_{t}+c\left(\xi_{x}-\tau_{t}\right)-c^{2} \tau_{x}\right) c^{2}+K\left(\xi_{t}+c\left(\xi_{x}-\tau_{t}\right)-c^{2} \tau_{x}\right)+ \\
& \quad+K c\left(\xi_{x}-2 \tau_{t}-3 c \tau_{x}\right)+\left(\xi_{t t}+c\left(2 \xi_{t x}-\tau_{t t}\right)+c^{2}\left(\xi_{x x}-2 \tau_{t x}\right)-c^{3} \tau_{x x}\right) c^{2}+ \\
& \quad+\left(A c^{4}+B c^{3}+D c^{2}+E c+G\right)\left(\xi_{x}-2 \tau_{t}-3 c \tau_{x}\right) c^{2}=0 .
\end{aligned}
$$

Данный многочлен равен нулю для всех $c$. В частности, при $c=0$ получаем $K \xi_{t}=0$. Производная многочлена при $c=0$ тоже равна нулю: $K\left(2 \xi_{x}-3 \tau_{t}\right)=0$. Так как $K \neq 0$, мы имеем $\xi_{t}=0,2 \xi_{x}=3 \tau_{t}$. Продифференцируем второе равенство по $t$ и получим, что $\tau_{t t}=0$, т. е. $\tau=k(x)+t m(x)$. Отсюда $\xi=(3 / 2) \int m(x) d x+l$. Подставим эти формулы в (21), получим

$$
\begin{aligned}
\frac{m^{\prime}}{2} c^{2} & +\left(5 A c^{4}+4 B c^{3}+3 D c^{2}+2 E c+G\right) \frac{m}{2}+\left(k^{\prime \prime}+t m^{\prime \prime}\right) c^{3}+ \\
& +\left(-A c^{5}+D c^{3}+2 E c^{2}+3 G c+4 K\right)\left(k^{\prime}+t m^{\prime}\right)=0
\end{aligned}
$$


и приравняем к нулю коэффициенты при степенях $c$. Получается система из шести уравнений:

$$
\begin{gathered}
A\left(k^{\prime}+t m^{\prime}\right)=0, \quad A m=0, \quad 2 B m+\left(k^{\prime \prime}+t m^{\prime \prime}\right)+D\left(k^{\prime}+t m^{\prime}\right)=0, \\
3 D m+m^{\prime}+4 E\left(k^{\prime}+t m^{\prime}\right)=0, \quad E m+3 G\left(k^{\prime}+t m^{\prime}\right)=0, \quad G m+8 K\left(k^{\prime}+t m^{\prime}\right)=0 .
\end{gathered}
$$

Если $A \neq 0$, то $m=0, k=$ const, и алгебра двумерна для любой функции вида (20). Таким образом, считаем, что $A=0$.

Последнее из шести уравнений системы - это многочлен по $t$, тождественно равный нулю, поэтому (так как $K \neq 0$ ) его коэффициенты нулевые: мы имеем $m=$ const и $k=-(G m / 8 K) x+k_{1}$. Подставим полученные формулы для $k$ и $m$ в оставшиеся уравнения системы:

$$
2 B m=\frac{D G m}{8 K}, \quad 3 D m=\frac{4 E G m}{8 K}, \quad E m=\frac{3 G^{2} m}{8 K}, \quad A\left(\frac{5 m}{2}+\frac{G m}{8 K}\right)=0 .
$$

Если $m=0$, то все эти соотношения выполнены для любой функции вида $\mathcal{F}(c)=$ $B c^{3}+D c^{2}+E c+G+K / c$, но алгебра двумерна. Если же $m \neq 0$, то

$$
E=\frac{3 G^{2}}{8 K}, \quad D=\frac{E G}{6 K}=\frac{G^{3}}{16 K^{2}}, \quad B=\frac{D G}{16 K}=\frac{G^{4}}{256 K^{3}} .
$$

Подставим полученные выражения для коэффициентов в $\mathcal{F}(c)$ и упростим. Получим, что для

$$
\mathcal{F}(c)=\frac{K}{c}\left(1+\frac{G c}{4 K}\right)^{4}=\frac{K}{c}(1+M c)^{4}
$$

алгебра симметрий трехмерна и имеет вид $\tau=-M m x+2 m t+k_{1}, \xi=3 m x+l$.

Полученная функция $\mathcal{F}$ на самом деле эквивалентна степенной функции, рассмотренной в лемме 1 . Чтобы убедиться в этом, воспользуемся уже упомянутой заменой $\bar{t}=x, \bar{x}=t, \bar{c}=1 / c$ вида 3 и приведем $\mathcal{F}$ к виду $\mathcal{F}=K(c+M)^{4}$, а затем заменой переменных $\bar{t}=t, \bar{x}=x+M t, \bar{c}=c+M$ вида 1 преобразуем ее в функцию $\mathcal{F}=K c^{4}$. Лемма доказана.

Лемма 3. Для функиий вида $\mathcal{F}(c)=(c-a)^{5} / c^{2}$ c a $\neq 0$ алгебра симметрий трехмерна и ее базис есть $\Xi_{1}=\partial_{t}, \Xi_{2}=\partial_{x}, \Xi_{3}=(3 a t+x) \partial_{t}+4 a x \partial_{x} ;$ уравнение с этой функиией эквивалентно уравнению со степенной функиией $\mathcal{F}(c)$.

ДокАзАТЕЛЬСтво. Подставим функцию $\mathcal{F}(c)$ заданного вида в уравнение $(17)$ и умножим его на $c^{3}$, получим

$$
\begin{aligned}
& -5 c(c-a)^{4}\left(\xi_{t}+c\left(\xi_{x}-\tau_{t}\right)-c^{2} \tau_{x}\right)+2(c-a)^{5}\left(\xi_{t}+c\left(\xi_{x}-\tau_{t}\right)-c^{2} \tau_{x}\right)+ \\
& \quad+(c-a)^{5}\left(\xi_{x}-2 \tau_{t}-3 c \tau_{x}\right) c+\left(\xi_{t t}+c\left(2 \xi_{t x}-\tau_{t t}\right)+c^{2}\left(\xi_{x x}-2 \tau_{t x}\right)-c^{3} \tau_{x x}\right) c^{3}=0 .
\end{aligned}
$$

При $c=0$ имеем $a^{5} \xi_{t}=0$. Поскольку по условиям леммы $a \neq 0$, мы выводим, что $\xi=\xi(x)$. Продифференцируем уравнение $(22)$ по $c$ и снова подставим $c=0$, это дает $3 \xi_{x}=4 \tau_{t}$, откуда $\tau=3 \xi_{x} t / 4+k(x)$. Подставим найденные $\xi$ и $\tau$ в $(22)$ и упростим, 
получим

$$
\begin{aligned}
5(c & -a)^{4}\left(c \frac{\xi_{x}}{4}-c^{2} \frac{3 \xi_{x x} t}{4}-c^{2} k_{x}\right)-2(c-a)^{5}\left(\frac{\xi_{x}}{4}-c \frac{3 \xi_{x x} t}{4}-c k_{x}\right)+ \\
& +(c-a)^{5}\left(\frac{\xi_{x}}{2}+c \frac{9 \xi_{x x} t}{4}+3 c k_{x}\right)+\left(c^{2} \frac{\xi_{x x}}{2}+c^{3} \frac{3 \xi_{x x x} t}{4}+c^{3} k_{x x}\right) c^{2}=0 .
\end{aligned}
$$

Это многочлен по $t$, его коэффициенты равны нулю:

$$
\begin{gathered}
(c-a)^{4}(3 c+2 a)\left(\frac{\xi_{x}}{4}-c k_{x}\right)+(c-a)^{5}\left(\frac{\xi_{x}}{2}+3 c k_{x}\right)+\left(c^{2} \frac{\xi_{x x}}{2}+c^{3} k_{x x}\right) c^{2}=0, \\
5 a(c-a)^{4} \xi_{x x}-c^{4} \xi_{x x x}=0 .
\end{gathered}
$$

Из последнего уравнения при $c=0$ получаем $\xi=n x+l$. Тогда из первого уравнения при $c=a$ мы имеем $k=k_{1} x+k_{2}$ и $n=4 a k_{1}$. В итоге с точностью до замен переменных из группы эквивалентности алгебра симметрий $\tau=k_{1}(3 a t+x)+k_{2}$, $\xi=4 k_{1} a x+l$ трехмерна.

Остается отметить, что заменой вида 3 наша функция приводится к функции $\mathcal{F}(c)=(a c-1)^{5}$, а последующей заменой вида 1 - к степенной функции с показателем 5. Лемма доказана.

Лемма 4. Для функиий вида

$$
\mathcal{F}(c)=A \exp \int \frac{3 c+a}{c^{2}+b c+d} d c, \quad A \neq 0,
$$

в случае, когда $\mathcal{F}(c)$ не является функиией из лемм 1-3, алгебра симметрий трехмерна и ее базис есть $\Xi_{1}=\partial_{t}, \Xi_{2}=\partial_{x}, \Xi_{3}=(x+(a-b) t) \partial_{t}+((a-2 b) x-d t) \partial_{x}$.

ДокАЗАТЕЛЬство. Воспользуемся тем, что $\left(c^{2}+b c+d\right) \mathcal{F}_{c}=\mathcal{F}(3 c+a)$ и подставим это соотношение в уравнение (17), получим

$$
\begin{aligned}
\mathcal{F}\left(-(a+3 c)\left(\xi_{t}\right.\right. & \left.\left.+c\left(\xi_{x}-\tau_{t}\right)-c^{2} \tau_{x}\right)+\left(\xi_{x}-2 \tau_{t}-3 c \tau_{x}\right)\left(d+b c+c^{2}\right)\right)+ \\
& +\left(\xi_{t t}+c\left(2 \xi_{t x}-\tau_{t t}\right)+c^{2}\left(\xi_{x x}-2 \tau_{t x}\right)-c^{3} \tau_{x x}\right)\left(d+b c+c^{2}\right)=0 .
\end{aligned}
$$

Возможны два случая.

1. Если множитель при $\mathcal{F}$ ненулевой, то $\mathcal{F}$ - отношение многочленов, причем многочлен в числителе имеет степень не выше пятой, а в знаменателе - не выше второй. Это возможно, только если знаменатель подынтегрального выражения для функции $\mathcal{F}$ имеет два действительных и различных корня (иначе $\mathcal{F}$ будет трансцедентной функцией). Найдем $\mathcal{F}$, предположив, что $c^{2}+b c+d=(c-m)(c-n)$ :

$$
\mathcal{F}=A(c-n)^{\frac{a+3 n}{n-m}}(c-m)^{-\frac{(a+3 m)}{n-m}} .
$$

Так как корни различны, степени многочленов должны быть целыми. Сумма степеней равна 3 , поэтому возможны только три варианта: степень одного из многочленов равна 0 , а другого равна 3 (тогда $b=(2 a / 3)+1, d=a(a+3) / 9$ или $b=(2 a / 3)-1$, $d=a(a-3) / 9)$; степень одного из многочленов равна -1 , а другого равна 4 (тогда $b=-a-5 n, d=n(a+4 n), n \neq-a / 3)$; степень одного из многочленов равна -2 , 
а другого равна 5 (тогда $b=-(a+7 n) / 2, d=n(a+5 n) / 2, n \neq-a / 3)$. При таких соотношениях степеней алгебра уже была нами изучена в леммах 1-3 (последний случай сводится к описанному в лемме 3 заменой $\bar{t}=t, \bar{x}=x-n t, \bar{c}=c-n)$.

2. Пусть коэффициент при $\mathcal{F}$ равен нулю, т. е.

$$
(3 c+a)\left(\xi_{t}+c\left(\xi_{x}-\tau_{t}\right)-c^{2} \tau_{x}\right)=\left(\xi_{x}-2 \tau_{t}-3 c \tau_{x}\right)\left(c^{2}+b c+d\right) .
$$

Раскрытие скобок и приравнивание к нулю коэффициентов при одинаковых степенях $c$ дает нам уравнения

$$
2 \xi_{x}-(a-3 b) \tau_{x}=\tau_{t}, \quad 3 \xi_{t}+(a-b) \xi_{x}=(a-2 b) \tau_{t}-3 d \tau_{x}, \quad a \xi_{t}=d \xi_{x}-2 d \tau_{t} .
$$

Выразим производные функции $\xi$ через производные функции $\tau$ :

$\xi_{x}=\frac{(a-3 b) \tau_{x}+\tau_{t}}{2}, \quad \xi_{t}=\frac{a-3 b}{6}\left((b-a) \tau_{x}+\tau_{t}\right)-d \tau_{x}, \quad a \xi_{t}=\frac{d(a-3 b) \tau_{x}-3 d \tau_{t}}{2}$.

Из второго и третьего равенства можно найти $a \xi_{t}$ и приравнять полученные выражения друг к другу. Приведя к общему знаменателю и группируя, получим уравнение $(a(a-3 b)+9 d)\left((b-a) \tau_{x}+\tau_{t}\right)=0$.

Учитывая, что $a(a-3 b)+9 d \neq 0$ (иначе $\mathcal{F}$ - многочлен), получаем $(b-a) \tau_{x}+\tau_{t}=0$, т. е. $\tau=\tau(x+(a-b) t)$. В этом случае соотношения для производных принимают вид $\xi_{x}=(a-2 b) \tau_{x}, \xi_{t}=-d \tau_{x}$.

Из условия совместности следует, что должно быть выполнено уравнение

$$
((a-2 b)(a-b)+d) \tau_{x x}=0 .
$$

Если равен нулю коэффициент при $\tau_{x x}$, то знаменатель в подынтегральном выражении, задающем $\mathcal{F}$ в условии леммы, имеет два различных корня и $\mathcal{F}(c)$ - многочлен. Если же $(a-2 b)(a-b)+d \neq 0$, то $\tau_{x x}=0$. Тогда $\tau_{t x}=(a-b) \tau_{x x}=0$ и $\tau_{t t}=(a-b)^{2} \tau_{x x}=0$. В силу данных соотношений $\tau=m(x+(a-b) t)+k$, $\xi=m((a-2 b) x-d t)+l$.

Вернемся к соотношению (17). Если $m=0$, то для всех $\mathcal{F}$ получается двумерная алгебра сдвигов. Если $m \neq 0$, то уравнение превращается в тривиальное тождество $0=0$, а алгебра $\tau=m(x+(a-b) t)+k, \xi=m((a-2 b) x-d t)+l$ оказывается трехмерной. Лемма доказана.

Лемма 5. Для функиий вида $\mathcal{F}(c)=A c^{a}$ c $A \neq 0, a \neq-1,0, \ldots, 5$ алгебра симметрий трехмерна и ее базис есть $\Xi_{1}=\partial_{t}, \Xi_{2}=\partial_{x}, \Xi_{3}=t \partial_{t}+\left((a-2)(a-1)^{-1} x\right) \partial_{x}$.

ДокАзАтЕльство. Подставим $\mathcal{F}(c)$ заданного вида в уравнение $(17)$, получим

$$
\begin{aligned}
a A c^{a-1}\left(\xi_{t}+c\left(\xi_{x}-\tau_{t}\right)-c^{2} \tau_{x}\right) & -A c^{a}\left(\xi_{x}-2 \tau_{t}-3 c \tau_{x}\right)-\xi_{t t}- \\
& -c\left(2 \xi_{t x}-\tau_{t t}\right)-c^{2}\left(\xi_{x x}-2 \tau_{t x}\right)+c^{3} \tau_{x x}=0 .
\end{aligned}
$$

Мы имеем многочлен по переменной $c$, тождественно равный нулю. Следовательно, все его коэффициенты нулевые, в том числе коэффициенты при $c^{a+1}$ и $c^{a}$, в результате $\tau_{x}=0$ и $(a-2) \tau_{t}=(a-1) \xi_{x}$. Отсюда $\tau=\tau(t)$ и $\xi=(a-2)(a-1)^{-1} \tau_{t} x+n(t)$.

Подставим полученные формулы для $\tau$ и $\xi$ снова в (17) и при $a \neq-1,0, \ldots, 5$ приравняем к нулю коэффициенты при оставшихся степенях $c$, откуда найдем, что 
$\tau=m t+k$ и $\xi=m(a-2)(a-1)^{-1} x+n$. Полученная алгебра трехмерна для любой функции $\mathcal{F}(c)=A c^{a}$ при $a \neq-1, \ldots, 5$. Заметим, что случаи $a=0,1, \ldots, 5$ уже рассмотрены в лемме 1 , а случай $a=-1$ рассмотрен в лемме 2. Лемма доказана.

Лемма 6. Для функиий вида $\mathcal{F}(c)=A e^{a c}$ c $A \neq 0, a \neq 0$ алгебра симметрий трехмерна и ее базис есть $\Xi_{1}=\partial_{t}, \Xi_{2}=\partial_{x}, \Xi_{3}=t \partial_{t}+(x-t / a) \partial_{x}$.

ДокАзАтЕльство. Воспользуемся тем, что $\mathcal{F}_{c}=a \mathcal{F}$, и подставим это соотношение в уравнение (17), получим

$$
\begin{aligned}
& \mathcal{F}\left(\xi_{x}-2 \tau_{t}-a \xi_{t}-c\left(a \xi_{x}-a \tau_{t}+3 \tau_{x}\right)+c^{2} a \tau_{x}\right)+ \\
& \quad+\xi_{t t}+c\left(2 \xi_{t x}-\tau_{t t}\right)+c^{2}\left(\xi_{x x}-2 \tau_{t x}\right)-c^{3} \tau_{x x}=0
\end{aligned}
$$

Так как функция $e^{a c}$ не может быть представлена в виде отношения многочленов и $A \neq 0, a \neq 0$, мы имеем $\tau=\tau(t), \xi=\tau_{t} x+l(t)$ и уравнения $\tau_{t}+a \xi_{t}=0, \xi_{t t}=0$, $2 \xi_{t x}-\tau_{t t}=0$. Из первого уравнения $\tau=k t+m, l=-k t / a+l_{1}$. Второе и третье уравнения выполнены, т. е. алгебра $\tau=k t+m, \xi=k x-k t / a+l_{1}$ трехмерна. Лемма доказана.

\section{5. Доказательство точной двумерности алгебры симметрий в осталь-} ных случаях. Поскольку для $\mathcal{F}=\mathcal{F}(c)$ уравнение $(13)$ выполняется при любых постоянных $\tau, \xi$, алгебра симметрий содержит двумерную коммутативную алгебру, и для завершения рассуждений с алгебрами, содержащими двумерную коммутативную подалгебру, нам необходимо показать, что в случаях, отличных от рассмотренных в леммах предыдущего пункта, алгебра симметрий имеет размерность, в точности равную двум. Для этого предположим, что уравнение (17) выполнено при некоторых непостоянных функциях $\tau(t, x), \xi(t, x)$. Подставим их в уравнение, которое теперь будем рассматривать как уравнение относительно $\mathcal{F}$ :

$$
-\mathcal{F}_{c} A+\mathcal{F} B+D=0 .
$$

1. Предположим сначала, что $A=0$. Тогда имеем $\tau_{x}=\xi_{t}=0$ и $\tau_{t}=\xi_{x}=$ const, причем эта константа отлична от нуля в силу предположения о том, что хотя бы одна из функций $\tau, \xi$ не является постоянной. Но тогда оказывается, что $B$ равна этой константе, $D=0$, следовательно, $\mathcal{F}=0$, и алгебра симметрий для этого случая уже найдена в лемме 1.

2. Пусть теперь $A \neq 0$. Дифференцируя соотношение (24) по $x$ и по $t$, получим систему из трех уравнений относительно $\mathcal{F}$ и $\mathcal{F}_{c}$ :

$$
-\mathcal{F}_{c} A+\mathcal{F} B+D=0, \quad-\mathcal{F}_{c} A_{x}+\mathcal{F} B_{x}+D_{x}=0, \quad-\mathcal{F}_{c} A_{t}+\mathcal{F} B_{t}+D_{t}=0 .
$$

Возможны три случая: система (25) несовместна, все три уравнения эквивалентны между собой, среди уравнений есть хотя бы два непропорциональных друг другу. Первый случай нас интересовать далее не будет, так как функций $\mathcal{F}(c)$, удовлетворяющих такой системе, не существует. Рассмотрим оставшиеся два случая.

2.1. Пусть $B A_{x}=B_{x} A$ и $B A_{t}=B_{t} A$, тогда функция $B$ пропорциональна $A$ с коэффициентом, зависящим только от $c$ :

$$
\phi(c)\left(\xi_{t}+c\left(\xi_{x}-\tau_{t}\right)-c^{2} \tau_{x}\right)=\xi_{x}-2 \tau_{t}-3 c \tau_{x} .
$$


2.1.1. Пусть $\tau_{x} \not \equiv 0$, тогда, фиксируя соответствующие значения $(t, x)$, получаем

$$
\phi(c)=\frac{3 c+a}{c^{2}+b c+d}
$$

где $a, b, d$ - некоторые константы. Подставим полученное выражение в (26)

$$
(3 c+a)\left(\xi_{t}+c\left(\xi_{x}-\tau_{t}\right)-c^{2} \tau_{x}\right)=\left(\xi_{x}-2 \tau_{t}-3 c \tau_{x}\right)\left(c^{2}+b c+d\right) .
$$

Раскроем скобки, коэффициенты при одинаковых степенях $c$ приравняем, выразим производные функции $\xi$ через производные функции $\tau$, получим

$$
\xi_{x}=\frac{(a-3 b) \tau_{x}+\tau_{t}}{2}, \quad \xi_{t}=\frac{(a-3 b)}{6}\left((b-a) \tau_{x}+\tau_{t}\right)-d \tau_{x}, \quad a \xi_{t}=\frac{d(a-3 b) \tau_{x}-3 d \tau_{t}}{2} .
$$

Из второго и третьего равенств можно найти $a \xi_{t}$ и приравнять полученные выражения друг к другу. Приведя к общему знаменателю и группируя, получим следующее уравнение:

$$
(a(a-3 b)+9 d)\left((b-a) \tau_{x}+\tau_{t}\right)=0 .
$$

2.1.1а. Предположим сначала, что $a(a-3 b)+9 d=0$. Если $3 b=a$ и, как следствие, $d=0$, то $2 \xi_{x}=\tau_{t}, 3 \xi_{t}+2 b \xi_{x}=b \tau_{t}$ и $a \xi_{t}=0$. Вычитая из второго из этих уравнений первое, умноженное на $b$, получаем, что $\xi_{t}=0$, откуда следует третье уравнение. В итоге мы имеем $\xi=\xi(x)$ и $\tau=2 \xi_{x} t+C(x)$. Подставим эти формулы в уравнение (17), получим

$$
c \mathcal{F}_{c}\left(\xi_{x}+2 c \xi_{x x} t+c C_{x}\right)-3 \mathcal{F}\left(\xi_{x}+2 c \xi_{x x} t+c C_{x}\right)-3 c^{2} \xi_{x x}-2 c^{3} \xi_{x x x} t-c^{3} C_{x x}=0 .
$$

В левой части стоит многочлен по $t$, приравняем к нулю его коэффициенты:

$$
c \mathcal{F}_{c} \xi_{x x}-3 \mathcal{F} \xi_{x x}-c^{2} \xi_{x x x}=0, \quad c \mathcal{F}_{c}\left(\xi_{x}+c C_{x}\right)-3 \mathcal{F}\left(\xi_{x}+c C_{x}\right)-3 c^{2} \xi_{x x}-c^{3} C_{x x}=0 .
$$

Если коэффициенты при $\mathcal{F}_{c}$ в обоих уравнениях равны нулю, то $\xi=$ const, $C=$ const и алгебра двумерна. Если хоть один из них ненулевой, то решение уравнения имеет вид $\mathcal{F}=M c^{3}-P c^{2}$, что уже рассмотрено в лемме 1.

Если $3 b \neq a$, то сделаем замену $\bar{t}=t, \bar{x}=x-(3 b-a) t / 3, \bar{c}=c-(3 b-a) / 3$. При такой замене мы имеем $\phi(c)=3 / c$, а соотношение (17) с новыми функциями $\bar{\tau}(\bar{t}, \bar{x})=\tau(t, x), \bar{\xi}(\bar{t}, \bar{x})=\xi-(3 b-a) \tau / 3$ перейдет в себя. С точностью до замен из алгебры эквивалентности снова получаем $\mathcal{F}=M c^{3}-P c^{2}$.

2.1.1б. Теперь предположим, что $a(a-3 b)+9 d \neq 0,(b-a) \tau_{x}+\tau_{t}=0$, т. е. $\tau=\tau(x+(a-b) t)$. Тогда $\xi_{x}=(a-2 b) \tau_{x}, \xi_{t}=-d \tau_{x}$. Из условия совместности получаем, что должно быть выполнено уравнение $((a-2 b)(a-b)+d) \tau_{x x}=0$. Если равен нулю коэффициент при $\tau_{x x}$, то $\xi=(a-2 b) \tau(x+(a-b) t)+C$. Чтобы упростить запись, сделаем замену $k=a-b$, тогда $\tau=\tau(x+k t), \xi=(k-b) \tau(x+k t)+C$. Подставим полученные выражения в уравнение (17), получим

$$
\begin{gathered}
\mathcal{F}_{c}\left(d+c b+c^{2}\right) \tau^{\prime}-\mathcal{F}(a+3 c) \tau^{\prime}-(a-b+c)\left(d+c b+c^{2}\right) \tau^{\prime \prime}=0, \\
\mathcal{F}=\exp \left(\int \frac{a+3 c}{(c-a+2 b)(c-b+a)} d c\right)\left(M+\int Q(a-b+c) e^{-\int \frac{(a+3 c)}{(c-a+2 b)(c-b+a)} d c} d c\right) .
\end{gathered}
$$


Сделаем замену переменных вида $\bar{t}=t, \bar{x}=x+(a-b) t, \bar{c}=c+a-b$. Эта замена принадлежит группе эквивалентности и переводит функцию $\mathcal{F}$ в функцию вида $\mathcal{F}(c)=(M(c+a-b)-Q) c(c+a-b)$, т. е. $\mathcal{F}-$ многочлен, что рассмотрено в лемме 1.

Если $(a-2 b)(a-b)+d \neq 0$, то $\tau_{x x}=0$. Тогда имеем уравнения $\tau_{t x}=(a-b) \tau_{x x}=0$ и $\tau_{t t}=(a-b)^{2} \tau_{x x}=0$, в силу которых $\tau=m(x+(a-b) t)+K, \xi=m((a-2 b) x-d t)+C$.

Вернемся к соотношению (17). Если $m=0$, то для всех $\mathcal{F}$ получается двумерная алгебра сдвигов. Если $m \neq 0$, уравнение упрощается до $\mathcal{F}_{c}\left(d+b c+c^{2}\right)-\mathcal{F}(a+3 c)=0$. Решая его, находим

$$
\mathcal{F}=M \exp \int \frac{a+3 c}{d+b c+c^{2}} d c .
$$

Алгебра симметрий для такой функции $\mathcal{F}$ изучена в лемме 4 .

2.1.2. Пусть $\tau_{x} \equiv 0, \xi_{t} \not \equiv 0$. Тогда из уравнения (26), фиксируя значения $(t, x)$, получаем, что $\phi(c)=a /(1+b c)$ и выполнено соотношение

$$
a\left(\xi_{t}+c\left(\xi_{x}-\tau_{t}\right)\right)=\left(\xi_{x}-2 \tau_{t}\right)(1+b c)
$$

Приравняем к нулю коэффициенты при степенях $c$ :

$$
(b-a) \xi_{x}+(a-2 b) \tau_{t}=0, \quad a \xi_{t}=\xi_{x}-2 \tau_{t} .
$$

Из второго уравнения имеем $2 \tau_{t}=\xi_{x}-a \xi_{t}$; после подстановки этого соотношения в первое уравнение получаем $a\left(\xi_{x}+(a-2 b) \xi_{t}\right)=0$.

2.1.2а. В случае $a=0$ получается, что $\tau=\tau(t), \xi=2 \tau_{t} x+C(t)$. Подставим эти соотношения в уравнение (17), получим

$$
-\mathcal{F}_{c}\left(2 \tau_{t t} x+C_{t}+c \tau_{t}\right)+2 \tau_{t t t} x+C_{t t}+3 c \tau_{t t}=0 .
$$

В левой части стоит многочлен по $x$, тождественно равный нулю, отсюда мы имеем уравнения $\mathcal{F}_{c} \tau_{t t}=\tau_{t t t}$ и $\mathcal{F}_{c}\left(C_{t}+c \tau_{t}\right)=C_{t t}+3 c \tau_{t t}$. В силу того что $\mathcal{F}=\mathcal{F}(c)$, из этих двух уравнений получаем $\mathcal{F}=K c+M$; этот случай уже был исследован в лемме 1 .

2.1.2б. В случае $a \neq 0$ получается, что $\tau_{t}=(b-a) \xi^{\prime}, \xi=\xi(t+(2 b-a) x)$. Так как $\tau=\tau(t)$, то $(b-a)(2 b-a) \xi^{\prime \prime}=0$.

2.1.2б(1). В случае $b=a$ получается что $\tau=C, \xi=\xi(t+b x)$, и мы имеем

$$
-\mathcal{F}_{c}(1+c b) \xi^{\prime}+b \mathcal{F} \xi^{\prime}+(1+c b)^{2} \xi^{\prime \prime}=0 .
$$

Если $\xi^{\prime}=0$, то алгебра двумерна, иначе разделение переменных в данном уравнении дает $\xi^{\prime \prime}=K \xi^{\prime}$ и $\mathcal{F}=(1+c b)(K c+M+B)$, т. е. $\mathcal{F}-$ многочлен, и этот случай рассмотрен в лемме 1 .

$2.1 .26(2)$. В случае $2 b=a$ получается $\tau=-b \xi(t)+C, \xi=\xi(t)$, и мы имеем

$$
-\mathcal{F}_{c}(1+c b) \xi_{t}+2 b \mathcal{F} \xi_{t}+(1+c b) \xi_{t t}=0 .
$$

Если алгебра не является двумерной, то разделение переменных дает по-прежнему $\xi^{\prime \prime}=K \xi^{\prime}$, в результате $\mathcal{F}(c)$ оказывается квадратным трехчленом.

$2.1 .2 б(3)$. Пусть $\xi^{\prime \prime}=0$, т. е. $\tau=(b-a) m t+k, \xi=m(t+(2 b-a) x)+l$. Подставим эти равенства в уравнение $(17)$, получим $m\left[\mathcal{F}_{c}(1+c b)-\mathcal{F} a\right]=0$. Случай $m=0$ отвечает двумерной алгебре. Если $m \neq 0$, то при $b \neq 0$ получаем $\mathcal{F}=M(1+c b)^{a / b}$ (что 
заменой переменных вида 1 можно привести к рассмотренной в лемме 5 функции $\left.\mathcal{F}=M c^{a}\right)$. При $b=0$ функция сводится к $\mathcal{F}=P e^{a c}$, рассмотренной в лемме 6 .

2.1.3. Предположим, что $\tau_{x} \equiv 0, \xi_{t} \equiv 0$. Мы находимся в рамках предположения, что в (24) $A \not \equiv 0$, при этом $A$ - это левая часть уравнения (26). Тогда должно быть выполнено условие $\xi_{x}-\tau_{t} \not \equiv 0$, и мы с учетом $(26)$ получаем, что $\phi(c)=a / c$, следовательно, $(a-1) \xi_{x}=(a-2) \tau_{t}=$ const.

Если $a=2$, то $\xi=l, \tau=\tau(t) \neq$ const. Подставим эти соотношения в уравнение (17), получим $c \mathcal{F}_{c} \tau_{t}-2 \mathcal{F} \tau_{t}-c \tau_{t t}=0$. Поскольку $\tau_{t} \neq 0$, мы имеем $\tau_{t t}=Q \tau_{t}$ и $\mathcal{F}=D c^{2}-Q c$. Алгебра симметрий для такой функции $\mathcal{F}$ исследована в лемме 1.

Аналогично, если $a=1$, то $\tau=b, \xi=\xi(x)$; подставим эти соотношения в уравнение $(17)$, получим $c \mathcal{F}_{c} \xi_{x}-\mathcal{F} \xi_{x}-c^{2} \xi_{x x}=0$, и вновь $\mathcal{F}=D c^{2}-Q c$.

Если $a \neq 1$ и $a \neq 2$, то получается алгебра вида $\tau=(a-1) k t+b, \xi=(a-2) k x+l$, причем $k \neq 0$ (как было отмечено выше, $\left.\xi_{x}-\tau_{t} \not \equiv 0\right)$. Вернемся к начальному соотношению, которое теперь приобретает вид $k\left(c \mathcal{F}_{c}-a \mathcal{F}\right)=0$. Из него, очевидно, следует, что $\mathcal{F}=M c^{a}$, а этот случай изучен в леммах 1 и 5 .

2.2. Пусть среди уравнений системы (25) есть хотя бы два непропорциональных друг другу (одно из соотношений $B A_{x}=B_{x} A$ и $B A_{t}=B_{t} A$ не выполнено тождественно). Тогда мы сможем найти анзац для $\mathcal{F}(c)$ : так как $A \neq 0$, из соответствующей пары уравнений при некоторых фиксированных значениях $(t, x)$ мы получаем, что $\mathcal{F}$ - это частное двух многочленов (в числителе стоит многочлен пятой степени, в знаменателе - второй). И, так как $B \neq 0$, из той же пары уравнений при соответствующих значениях $(t, x)$ получаем, что $\mathcal{F}_{c}$ - это частное двух многочленов (в числителе стоит многочлен четвертой степени, в знаменателе - второй). Дифференцируя выражение для $\mathcal{F}$ по $c$ и сравнивая с видом $\mathcal{F}_{c}$, получаем, что возможны только два случая: в знаменателе обеих функций стоит константа или после выделения целой части дробь сокращается, т. е. возможны следующие $\mathcal{F}$ :

$$
\begin{aligned}
& \mathcal{F}(c)=Q c^{5}+A c^{4}+B c^{3}+D c^{2}+E c+G, \\
& \mathcal{F}(c)=A c^{4}+B c^{3}+D c^{2}+E c+G+\frac{K}{c+M} .
\end{aligned}
$$

Алгебры, соответствующие этим функциям, исследованы в леммах 1 и 2 .

Таким образом, рассмотрены все возможные фукции $\mathcal{F}(c)$, которым соответствует алгебра, содержащая двумерную коммутативную подалгебру. Мы показали, что все случаи, когда эта алгебра шире, чем двумерная, уже учтены в леммах 1-5 и выписаны в табл. 1 и 2.

\section{6. Уравнения с двумерными некоммутативными алгебрами симмет-}

рий. Пусть уравнению $(1)$ с некоторой функцией $\mathcal{F}(t, x, c)$ соответствует двумерная некоммутативная алгебра симметрий с базисом $\Xi_{1}=\tau_{1} \partial_{t}+\xi_{1} \partial_{x}, \Xi_{2}=\tau_{2} \partial_{t}+\xi_{2} \partial_{x}$. Тогда, как и в случае коммутативной алгебры, найдем такую замену переменных $\bar{t}=\varphi(t, x), \bar{x}=\psi(t, x)$ из группы эквивалентности, что в новых переменных будут выполнены равенства $\tau_{1}=0, \xi_{1}=1$. Из соотношения для коммутатора находим, что $\tau_{2}=\tau_{2}(t), \xi_{2}=-x+D(t)$. Затем заменой

$$
\varphi(t)=\exp \left(-\int \frac{t d t}{\tau_{2}(t)}\right), \quad \psi(x)=x+\psi_{1}(t), \quad\left(\psi_{1}\right)_{t} \tau+D(t)+\psi_{1}(t)=0,
$$


которая лежит в группе эквивалентности и не меняет $\left(\tau_{1}, \xi_{1}\right)$, приведем пару функций $\left(\tau_{2}, \xi_{2}\right)$ к виду $(-t,-x)$. Подставим это в соотношение $(13)$ и найдем, что данной группе отвечает функция $\mathcal{F}(t, c)=T(c) / t$. Определим, какие $\tau, \xi$ соответствуют функции $\mathcal{F}$ такого вида. Сначала выразим в $(13) \mathcal{F}(t, c)$ через $T(c)$ и приведем к общему знаменателю, получим

$$
\begin{aligned}
-T_{c} t\left(\xi_{t}+c \xi_{x}-c \tau_{t}-c^{2} \tau_{x}\right) & +T\left(t\left(\xi_{x}-2 \tau_{t}-3 c \tau_{x}\right)+\tau\right)+ \\
& +t^{2}\left(\xi_{t t}+2 c \xi_{t x}+c^{2} \xi_{x x}-c \tau_{t t}-2 c^{2} \tau_{t x}-c^{3} \tau_{x x}\right)=0 .
\end{aligned}
$$

Далее будем использовать уравнение (27) вместо (13) и вести рассуждения в терминах функции $T(c)$.

\section{7. Уравнения с большими алгебрами симметрий, содержащими дву-} мерную некоммутативную подалгебру. Как и в коммутативном случае, рассмотрим отдельно алгебры симметрий, соответствующие уравнению (1) с функциями $T(c)$ из некоторых классов. Все дальнейшие результаты формулируются с точностью до преобразований из группы эквивалентности.

В данном случае мы также будем использовать замены переменных, которые лежат в группе эквивалентности уравнений $(1),(3),(4)$ и позволяют "упрощать" функцию $\mathcal{F}(t, x, c)$ :

1) замена $\bar{t}=t, \bar{x}=x+a t, \bar{c}=c+a$ переводит $\mathcal{F}(t, x, c)$ в $\overline{\mathcal{F}}(\bar{t}, \bar{x}-a \bar{t}, \bar{c}-a)$;

2) замена $\bar{t}=t^{a+1} /(a+1), \bar{x}=x, \bar{c}=c t^{-a}$ переводит аффинное семейство функций $\mathcal{F}(t, c)=\left(M c^{q}+a c\right) / t$ в линейное семейство функций $\overline{\mathcal{F}}(\bar{t}, \bar{c})=M \bar{c}^{q}((a+1) \bar{t})^{\frac{(q-2) a-1}{a+1}} ;$

3) замена $\bar{t}=t, \bar{x}=x+(t-t \ln t) / m, \bar{c}=c-\ln t / m$ переводит $\mathcal{F}(t, c)=\frac{M e^{m c}}{t}+\frac{1}{m t}$ в $\overline{\mathcal{F}}(\bar{c})=M e^{m \bar{c}}$

4) замена $\bar{t}=x, \bar{x}=t, \bar{c}=1 / c$ переводит $\mathcal{F}(t, x, c)$ в $\overline{\mathcal{F}}(\bar{t}, \bar{x}, \bar{c})=-\bar{c}^{3} \mathcal{F}(\bar{x}, \bar{t}, 1 / \bar{c})$;

5) замена $\bar{t}=t, \bar{x}=x-a t^{2} / 2, \bar{c}=c-a t$ переводит $\mathcal{F}=a$ в $\overline{\mathcal{F}}=0$.

Лемма 7. Из функиий вида $T(c)=Q c^{5}+A c^{4}+B c^{3}+D c^{2}+E c+G$ алгебру симметрий размерности больше двух имеют те, которые можно свести с помощъю замены переменных $к$ описанным в лемме 1 , при этом функция $T(c)=B c^{3}-c / 2$ (соответствующая $\mathcal{F}=T(c) / t$ ) сводится $\kappa \mathcal{F}(x)=A / x^{3}$, для которой алгебра является трехмерной; ее базис есть $\Xi_{1}=\partial_{t}, \Xi_{2}=t \partial_{t}+(x / 2) \partial_{x}, \Xi_{3}=t^{2} \partial_{t}+t x \partial_{x}$.

ДокАзАТЕЛЬство. Подставим анзац $T(c)$ в $(27)$ :

$$
\begin{aligned}
-\left(5 Q c^{4}\right. & \left.+4 A c^{3}+3 B c^{2}+2 D c+E\right) t \xi_{t}- \\
& -\left(5 Q c^{5}+4 A c^{4}+3 B c^{3}+2 D c^{2}+E c\right)\left(t \xi_{x}-t \tau_{t}\right)+ \\
& +\left(2 Q c^{6}+A c^{5}-D c^{3}-2 E c^{2}-3 G c\right) t \tau_{x}+ \\
& +\left(Q c^{5}+A c^{4}+B c^{3}+D c^{2}+E c+G\right)\left(t \xi_{x}-2 t \tau_{t}+\tau\right)+ \\
& +t^{2}\left(\xi_{t t}+c\left(2 \xi_{t x}-\tau_{t t}\right)+c^{2}\left(\xi_{x x}-2 \tau_{t x}\right)-c^{3} \tau_{x x}\right)=0 .
\end{aligned}
$$


Приравняем к нулю коэффициенты при всех степенях $c$, получим уравнения

$$
\begin{gathered}
Q \tau_{x}=0, \quad Q\left(3 t \tau_{t}-4 t \xi_{x}+\tau\right)+A t \tau_{x}=0, \quad 5 Q t \xi_{t}+A\left(3 t \xi_{x}-2 t \tau_{t}-\tau\right)=0, \\
4 A t \xi_{t}+B\left(2 t \xi_{x}-t \tau_{t}-\tau\right)+D t \tau_{x}+t^{2} \tau_{x x}=0, \\
3 B t \xi_{t}+D\left(t \xi_{x}-\tau\right)+2 E t \tau_{x}-t^{2}\left(\xi_{x x}-2 \tau_{t x}\right)=0, \\
2 D t \xi_{t}+E\left(t \tau_{t}-\tau\right)+3 G t \tau_{x}-t^{2}\left(2 \xi_{t x}-\tau_{t t}\right)=0 \\
E t \xi_{t}+G\left(-t \xi_{x}+2 t \tau_{t}-\tau\right)-t^{2} \xi_{t t}=0 .
\end{gathered}
$$

1. Предположим, что $Q \neq 0$. У многочлена пятой степени всегда есть вещественный корень; пусть $T(M)=0, M \in \mathbb{R}$. Сделаем замену переменных $\bar{t}=t$, $\bar{x}=x-M t, \bar{c}=c-M$ вида 1. Эта замена переводит уравнение с функцией $T(c)$ заданного в условии леммы вида в уравнение с $T=Q c^{5}+A c^{4}+B c^{3}+D c^{2}+E c$. Для такой $T(c)$ система уравнений записывается как

$$
\begin{gathered}
\tau_{x}=0, \quad 3 t \tau_{t}-4 t \xi_{x}+\tau=0, \quad 5 Q t \xi_{t}+A\left(3 t \xi_{x}-2 t \tau_{t}-\tau\right)=0 \\
4 A t \xi_{t}+B\left(2 t \xi_{x}-t \tau_{t}-\tau\right)=0, \quad 3 B t \xi_{t}+D\left(t \xi_{x}-\tau\right)-t^{2} \xi_{x x}=0 \\
2 D t \xi_{t}+E\left(t \tau_{t}-\tau\right)-t^{2}\left(2 \xi_{t x}-\tau_{t t}\right)=0, \quad E \xi_{t}-t \xi_{t t}=0
\end{gathered}
$$

Из первых двух уравнений получаем, что $\tau=\tau(t)$ и $\xi=\left(3 \tau_{t} / 4+\tau / 4 t\right) x+C(t)$. Из третьего, четвертого, пятого и шестого уравнений имеем $3 t \tau_{t t}+\tau_{t}-\tau / t=0$, т. е. $\tau=k t+m t^{-1 / 3}$ и $\xi=k x+C(t)$, кроме того, справедливы уравнения

$$
15 Q t^{4 / 3} C_{t}=A m, \quad 6 A t^{4 / 3} C_{t}=B m, \quad 3 B t^{4 / 3} C_{t}=D m, \quad 9 D t^{4 / 3} C_{t}=2 m(3 E-1) .
$$

Выразим $C$ из первого из этих уравнений, $C=-A m t^{-1 / 3} / 5 Q+C_{1}$, тогда остальные приобретут вид

$$
m\left(\frac{2 A^{2}}{5 Q}-B\right)=0, \quad m\left(\frac{B A}{5 Q}-D\right)=0, \quad m\left(\frac{3 D A}{5 Q}-2(3 E-1)\right)=0 .
$$

При $m=0$ получаем двумерную алгебру, поэтому далее считаем, что $m \neq 0$. Тогда

$$
B=\frac{2 A^{2}}{5 Q}, \quad D=\frac{B A}{5 Q}=\frac{2 A^{3}}{25 Q^{2}}, \quad E=\frac{A^{4}}{125 Q^{3}}+\frac{1}{3} .
$$

С учетом этих соотношений выражение для $T$ можно привести к виду

$$
T(c)=Q\left(c+\frac{A}{5 Q}\right)^{5}+\frac{1}{3}\left(c+\frac{A}{5 Q}\right)-\frac{A}{5 Q}\left(\frac{A^{4}}{5^{4} Q^{3}}+\frac{1}{3}\right) .
$$

Подставим $\tau$ и $\xi$ в последнее уравнение системы $(28)$ и получим $(3 E+4) A=0$. Если $A=0$, то $B=D=0, E=1 / 3, C=$ const и для $T(c)=Q c^{5}+c / 3$ алгебра симметрий $\tau=k t+m t^{-1 / 3}, \xi=k x+C_{1}$ трехмерна. Если $A \neq 0$, то

$$
E=-\frac{4}{3}, \quad T=Q\left(c+\frac{A}{5 Q}\right)^{5}+\frac{1}{3}\left(c+\frac{A}{5 Q}\right)
$$

и из (29) мы имеем $3 A^{4}+5^{4} Q^{3}=0$. После аналогичной замены мы получаем функцию $T(c)=Q c^{5}+c / 3$, и соответствующая алгебра симметрий имеет тот же 
вид, что и выше. Замена переменных вида 2 (с $q=5, a=1 / 3$ ) позволяет привести уравнение (1) к уравнению с $\mathcal{F}=Q c^{5}$.

2. Предположим, что $Q=0, A \neq 0$. Тогда $\tau=\tau(t)$ и $\xi=\left(2 \tau_{t} / 3+\tau / 3 t\right) x+C(t)$ и справедливы уравнения

$$
\begin{gathered}
12 A t \xi_{t}+B\left(t \tau_{t}-\tau\right)=0, \quad 9 B t \xi_{t}+2 D\left(t \tau_{t}-\tau\right)=0 \\
6 D t \xi_{t}+3 E\left(t \tau_{t}-\tau\right)-t^{2} \tau_{t t}-2 t \tau_{t}+2 \tau=0, \quad 3 E t \xi_{t}+4 G\left(t \tau_{t}-\tau\right)-3 t^{2} \xi_{t t}=0 .
\end{gathered}
$$

При подстановке $\xi$ в эти уравнения получим многочлены по $x$. Из первого уравнения следует, что $2 \tau_{t t}+\tau_{t} / t-\tau / t^{2}=0$, т. е. $\tau=k t+m t^{-1 / 2} \xi_{t}=k x+C(t)$. Упростим приведенные выше соотношения:

$$
\begin{aligned}
& 8 A C_{t}=B m t^{-3 / 2}, \quad 3 B C_{t}=D m t^{-3 / 2}, \\
& 8 D C_{t}=3 m t^{-3 / 2}(2 E-1), \quad E C_{t}=2 G m t^{-3 / 2}+t C_{t t} \text {. }
\end{aligned}
$$

Отсюда $C=-B m t^{-1 / 2} / 4 A+C_{1}$, и, так как $m=0$ приводит к двумерной алгебре, мы можем считать, что $m \neq 0$, следовательно, $D=3 B^{2} / 8 A, 2 E-1=D B / 3 A=B^{3} / 8 A^{2}$ и $6 G=3 B(2 E+3) / 16 A$. Таким образом,

$$
\tau=k t+m t^{-1 / 2}, \quad \xi=k x-\frac{B m t^{-1 / 2}}{4 A}+C_{1}
$$

есть трехмерная алгебра для функции $T=A(c+B / 4 A)^{4}+(c+B / 4 A) / 2$, которую заменой переменных из группы эквивалентности можно привести к виду $T=A c^{4}+c / 2$. В этом случае трехмерная алгебра симметрий имеет вид $\tau=k t+m t^{-1 / 2}, \xi=k x+C_{1}$. $\mathrm{C}$ помощью замены вида 2 соответствующее уравнение также приводится к уравнению с $\mathcal{F}=A c^{4}$.

3. Предположим, что $Q=A=0, B \neq 0$. Тогда мы получаем многочлен третьей степени, у которого всегда есть вещественный корень; пусть $T(M)=0, M \in \mathbb{R}$. Сделаем замену переменных $\bar{t}=t, \bar{x}=x-M t, \bar{c}=c-M$. Эта замена принадлежит группе эквивалентности и переводит $T$ в функцию вида $T=B c^{3}+D c^{2}+E c$. Тогда система принимает вид

$$
\begin{gathered}
B\left(2 t \xi_{x}-t \tau_{t}-\tau\right)+D t \tau_{x}+t^{2} \tau_{x x}=0, \\
3 B t \xi_{t}+D\left(t \xi_{x}-\tau\right)+2 E t \tau_{x}-t^{2}\left(\xi_{x x}-2 \tau_{t x}\right)=0, \\
2 D t \xi_{t}+E\left(t \tau_{t}-\tau\right)-t^{2}\left(2 \xi_{t x}-\tau_{t t}\right)=0, \quad E \xi_{t}-t \xi_{t t}=0 .
\end{gathered}
$$

3.1. Предположим, что $E \neq-1,-1 / 2,0$, тогда из двух последних уравнений системы находим

$$
\xi=n(x) \frac{t^{E+1}}{E+1}+l(x), \quad \tau=k(x) t^{-E}+m(x) t+n_{x} \frac{t^{E+2}}{(E+1)^{2}}-n(x) \frac{2 D t^{E+1}}{E(2 E+1)} .
$$

После подстановки этих функций во второе уравнение системы (30) имеем

$$
\begin{aligned}
D\left(l_{x}-m\right) t & +\left(2(E+1) m_{x}-l_{x x}\right) t^{2}-D k t^{-E}+\left(3 B+\frac{2 D^{2}}{E(2 E+1)}\right) n t^{E+1}- \\
& -\frac{D(E+2)(3 E+2)}{3 E(E+1)^{2}} n_{x} t^{E+2}+\frac{3}{E+1} n_{x x} t^{E+3}=0 .
\end{aligned}
$$


3.1.1. Если $E \neq-2,-3 / 2,1$, то степени переменной $t$ в уравнении (31) не дублируются, тогда, приравнивая к нулю коэффициенты при различных степенях, получаем систему

$$
\begin{gathered}
D\left(l_{x}-m\right)=0, \quad 2(E+1) m_{x}-l_{x x}=0, \quad D k=0, \\
n\left(3 B+\frac{2 D^{2}}{E(2 E+1)}\right)=0, \quad D n_{x}(3 E+2)=0, \quad n_{x x}=0 .
\end{gathered}
$$

3.1.1а. Пусть $D=0$, тогда $n=0$ и $l_{x}=2(E+1) m+l_{1}$. Подставим эти соотношения в первое из уравнений (30), получим

$$
2 B\left((2 E+1) m+l_{1}\right) t+B(E-1) k t^{-E}+k_{x x} t^{2-E}+m_{x x} t^{3}=0 .
$$

3.1.1a(1). Если $E=-3$, то $k=0, m=l_{1} / 5$, тогда $l=l_{1} x / 5+l_{2}$ и алгебра двумерна.

3.1.1a $(2)$. Если $E \neq-3$, то все степени переменной $t$ различны, и тогда $k=0$, $m=m_{1} x+m_{2}$ и $(2 E+1) m_{1} x+(2 E+1) m_{2}+l_{1}=0$. Так как $E \neq-1 / 2$, то $m_{1}=0$ и $l=m_{2} x+l_{2}$, и мы снова получаем двумерную алгебру.

3.1.1б. Пусть теперь $D \neq 0$, тогда $k=0, m=m_{2}, l=m_{2} x+l_{1}, n=n_{1} x+n_{2}$ и

$$
n\left(3 B+\frac{2 D^{2}}{E(2 E+1)}\right)=0, \quad n_{1}(3 E+2)=0 .
$$

3.1.1б(1). Если $n=0$, то алгебра симметрий $\tau=m_{2} t, \xi=m_{2} x+l_{1}$ двумерна, и последнее уравнение выполнено автоматически.

3.1.1б(2). Если $n \neq 0, n_{1}=0$, то $B=-2 D^{2} /(3 E(2 E+1))$ и

$$
\tau=m_{2} t-\frac{2 D n_{2} t^{E+1}}{E(2 E+1)}, \quad \xi=\frac{n_{2} t^{E+1}}{E+1}+m_{2} x+l_{1} .
$$

Подставим эти соотношения в первое уравнение системы $(30)$, получим $E=-2$, что невозможно.

3.1.1б(3). Пусть $n_{1} \neq 0, n_{2} \neq 0$ и $E=-2 / 3, B=-3 D^{2}$. Подставим эти условия в первое уравнение системы (30), получим

$$
D^{2} n_{1}\left(-\frac{3(E-1)}{(E+1)^{2}}-\frac{2}{E(2 E+1)}\right) t^{E+2}+2 B D \frac{E+2}{E(2 E+1)}\left(n_{1} x+n_{2}\right) t^{E+1}=0 .
$$

Отсюда следует, что $n_{1}=0$, и это противоречит сделанному предположению.

3.1.2. Рассмотрим оставшиеся случаи.

3.1.2а. При $E=-2$ из последних двух уравнений системы (30) находим

$$
\xi=-\frac{n(x)}{t}+l(x), \quad \tau=k(x) t^{2}+m(x) t+n_{x}-\frac{D n(x)}{3 t} .
$$

Уравнение (31) принимает вид

$$
\left(D\left(l_{x}-m\right)-3 n_{x x}\right) t-\left(2 m_{x}+l_{x x}+D k\right) t^{2}+\left(3 B+\frac{D^{2}}{3}\right) \frac{n}{t}=0 .
$$

Приравняем к нулю коэффициенты при степенях $t$ и получим два случая. 
$3.1 .2 \mathrm{a}(1)$. Если $D=0$, то $n=0$ и $l_{x}=-2 m+l_{1}$. Подставим эти соотношения в первое уравнение системы (30) и получим, что $k=0, m=l_{1} / 3, l=l_{1} x / 3+l_{2}$ есть двумерная алгебра.

$3.1 .2 \mathrm{a}(2)$. Если $D \neq 0$ и $n=0$, то $m=l_{x}$ и $k=-3 l_{x x} / D$. Подставим эти соотношения в первое уравнение системы (30) и получим

$$
\left(\frac{9 B}{D}+D\right) l_{x x} t^{2}-2 l_{x x x} t^{3}-\frac{3}{D} l_{x x x x} t^{4}=0 .
$$

Отсюда $l=l_{1} x^{2}+l_{2} x+l_{3}$ и либо $l_{1}=0$ (и алгебра двумерна), либо $B=-D^{2} / 9$ и $T(c)=-D^{2} c^{3} / 9+D c^{2}-2 c$. Заменой $\bar{t}=t, \bar{x}=x-3 t / d, \bar{c}=c-3 / D$ уравнение с такой функцией $T(c)$ приводится к случаю $T(c)=-D^{2} c^{3} / 9+c$. Далее можно снова воспользоваться заменой вида $2($ с $q=3, a=1)$, приводящей к $\mathcal{F}=M c^{3}$.

$3.1 .2 \mathrm{a}(3)$. Если $D \neq 0$ и $n \neq 0$, то $B=-D^{2} / 9$. Функцию такого вида мы рассмотрели выше.

3.1.2б. Если $E=-3 / 2$, то уравнение (31) имеет вид

$D\left(l_{x}-m\right) t+\left(-m_{x}-l_{x x}\right) t^{2}+\left(-6 n_{x x}-D k\right) t^{3 / 2}+\left(3 B+\frac{2 D^{2}}{3}\right) n t^{-1 / 2}-\frac{10 D}{3} n_{x} t^{1 / 2}=0$.

$3.1 .26(1)$. Пусть $D=0$, тогда $n=0$ и $l_{x}=-m+l_{1}$. Подставим эти соотношения в первое уравнение системы (30) и получим

$$
2 B\left(-2 m+l_{2}\right) t-\frac{5 B}{2} k(x) t^{3 / 2}+k_{x x} t^{7 / 2}+m_{x x} t^{3}=0 .
$$

Тогда $m=l_{1} / 2, k=0$ и $l=l_{1} x / 2+l_{2}$ есть двумерная алгебра.

$3.1 .26(2)$. Пусть теперь $D \neq 0$, тогда $n=n_{1}, k=0, m=m_{1}, l=m_{1} x+l_{1}$ и $n_{1}\left(9 B+2 D^{2}\right)=0$. Если $n_{1}=0$, то алгебра двумерна. Поэтому мы считаем, что $B=-2 D^{2} / 9$. В этом случае $\tau=m_{1} t-2 D n_{1} t^{-1 / 2} / 3, \xi=-2 n_{1} t^{-1 / 2}+m_{1} x+l_{1}$. Подставим эти формулы в последнее уравнение системы (30) и получим, что $n_{1}=0$; при этом алгебра двумерна.

3.1.2в. Если $E=1$, то уравнение (31) имеет вид

$$
D\left(l_{x}-m\right) t+\left(4 m_{x}-l_{x x}+3 B n+\frac{2 D^{2} n}{3}\right) t^{2}-D k t^{-1}-\frac{5 D}{4} n_{x} t^{3}+\frac{3}{2} n_{x x} t^{4}=0 .
$$

3.1.2в $(1)$. Пусть $D=0$, тогда $T(c)=B c^{3}+c$. Функция такого вида эквивалентна (с использованием замены вида 2) функции $\mathcal{F}=B c^{3}$.

$3.1 .2 \mathrm{~B}(2)$. Пусть $D \neq 0$, тогда $l_{x}=m, k=0, n=n_{1}$ и $l_{x x}=-n_{1}\left(B+2 D^{2} / 9\right)$, т. е.

$$
l=-n_{1}\left(B+\frac{2 D^{2}}{9}\right) \frac{x^{2}}{2}+l_{1} x+l_{2}, \quad m=-n_{1}\left(B+\frac{2 D^{2}}{9}\right) x+l_{1} .
$$

Подставим эти соотношения в первое из уравнений $(30)$, получим $n_{1}\left(B-2 D^{2} / 9\right)=0$. Если $n_{1}=0$, то алгебра двумерная, поэтому мы считаем, что $n_{1} \neq 0$, а тогда $B=2 D^{2} / 9$. Для функции $T(c)=2 D^{2} c^{3} / 9+D c^{2}+c$ алгебра симметрий

$$
\tau=\frac{2}{3} D n_{1} t\left(-\frac{2}{3} D x-t\right)+l_{1} t, \quad \xi=n_{1}\left(\frac{t^{2}}{2}-\frac{2}{9} D^{2} x^{2}\right)+l_{1} x+l_{2}
$$


трехмерна. Заменой переменных $\bar{t}=t, \bar{x}=x+3 t / 2 D, \bar{c}=c+3 / 2 D$ можно привести $T(c)$ к виду $T(c)=2 D^{2} c^{3} / 9-c / 2$. Это единственный случай, который не сводится к $\mathcal{F}(c)$, его мы выделили в формулировке леммы.

3.2.1. Пусть $E=-1$, из последнего и предпоследнего уравнений системы (30) получим $\xi=n(x) \ln t+l(x)$ и $\tau=k(x) t \ln t+m(x) t+n_{x} t \ln ^{2} t-2 D n(x) ;$ после подстановки во второе уравнение системы (30) имеем уравнение

$\left(3 B+2 D^{2}\right) n+D\left(l_{x}-m+4 n_{x}\right) t+\left(2 k_{x}-l_{x x}\right) t^{2}+D\left(n_{x}-k\right) t \ln t-D n_{x} t \ln ^{2} t+3 n_{x x} t^{2} \ln t=0$.

В итоге получаем систему

$$
\begin{gathered}
n\left(3 B+2 D^{2}\right)=0, \quad D\left(l_{x}-m+4 n_{x}\right)=0, \quad 2 k_{x}-l_{x x}=0, \\
D\left(n_{x}-k\right)=0, \quad D n_{x}=0, \quad n_{x x}=0 .
\end{gathered}
$$

3.2.1а. Пусть $D=0$, тогда $n=0$ и $l_{x}=2 k+l_{1}$. Подставим эти соотношения в первое уравнение системы (30), получим

$$
B\left(3 k+2 l_{1}-2 m\right) t-2 B k t \ln t+k_{x x} t^{3} \ln t+m_{x x} t^{3}=0 .
$$

Отсюда $m=l_{1}, k=0$ и $l=l_{1} x+l_{2}$ и алгебра двумерна.

3.2.1б. Пусть $D \neq 0$, тогда $n=n_{2}, k=0, l=l_{1} x+l_{2}, m=l_{1}$ и $\left(3 B+2 D^{2}\right) n=0$. Если $n=0$, то алгебра двумерна, иначе $B=-2 D^{2} / 3$, и в силу последнего уравнения системы $n_{2}=0$, а тогда алгебра снова двумерна.

3.2.2. Пусть $E=-1 / 2$, тогда $\xi=n(x) t^{1 / 2}+l(x)$; из третьего уравнения системы (30) найдем, что $\tau=k(x) t^{1 / 2}+m(x) t+2 n_{x} t^{3 / 2}+2 D n t^{1 / 2} \ln t$. Подставим это выражение во второе уравнение системы (30), получим

$$
\begin{aligned}
\left(\frac{3}{2} B n\right. & -D k) t^{1 / 2}+D\left(l_{x}-m\right) t+3 D n_{x} t^{3 / 2}+ \\
& +\left(m_{x}-l_{x x}\right) t^{2}+3 n_{x x} t^{5 / 2}-2 D^{2} n t^{1 / 2} \ln t=0 .
\end{aligned}
$$

3.2.2а. Пусть $D=0$, тогда $n=0$ и $l_{x}=m+l_{1}$. Подставим эти соотношения в первое уравнение системы (30), получим

$$
B\left(2 l_{1} t-\frac{3}{2} k t^{1 / 2}\right)+k_{x x} t^{5 / 2}+m_{x x} t^{3}=0 .
$$

Отсюда $k=0, l_{1}=0, m=m_{1} x+m_{2}$ и $l=m_{1} x^{2} / 2+m_{2} x+l_{2}$. Таким образом, для $T(c)=B c^{3}-c / 2$ алгебра симметрий $\tau=m_{1} x t+m_{2} t, \xi=m_{1} x^{2} / 2+m_{2} x+l_{2}$ трехмерна. Заметим, что функция, полученная в п. 3.1.2в $(2)$, совпадает с данной с точностью до переобозначений.

3.2.2б. Пусть $D \neq 0$, тогда $n=0, k=0, l_{x}=m$. Первое уравнение системы (30) в этом случае имеет вид $D m_{x}+t m_{x x}=0$, тогда $m=m_{2}, l=m_{2} x+l_{1}$ и алгебра двумерна.

3.2.3. Пусть $E=0$, тогда $\xi=n(x) t+l(x)$, и из третьего уравнения системы $(30)$ мы получаем $\tau=k(x) t+m(x)+n_{x} t^{2}-2 D n t \ln t$. Подставим эти соотношения во второе уравнение системы, получим

$-D m+\left(3 B n+D l_{x}-D k\right) t+\left(-l_{x x}+2 k_{x}-4 D n_{x}\right) t^{2}+3 n_{x x} t^{3}-4 D n_{x} t^{2} \ln t+2 D^{2} n t \ln t=0$. 
3.2.3а. Пусть $D=0$, тогда $n=0$ и $l_{x}=2 k+l_{1}$. Из первого уравнения системы (30) имеем $B\left(2\left(l_{1}+k\right) t-m\right)+\left(m_{x x}\right) t^{2}+t^{3} k_{x x}=0$. Тогда $k=-l_{1}, m=0, l=-l_{1} x+l_{2}$ и алгебра двумерна.

3.2.3б. Пусть $D \neq 0$, тогда $m=0, n=0, l_{x}=k=k_{2}$ и алгебра двумерна.

4. Если $Q=A=B=0, D \neq 0$, то заменой переменных $\bar{t}=t, \bar{x}=x+E t / 2 D$, $\bar{c}=c+E / 2 D$ приведем $T(c)$ к виду $T=D c^{2}+G$. Тогда система (28) упростится до

$$
\begin{gathered}
D \tau_{x}+t \tau_{x x}=0, \quad D\left(t \xi_{x}-\tau\right)-t^{2}\left(\xi_{x x}-2 \tau_{t x}\right)=0 \\
2 D \xi_{t}+3 G \tau_{x}-t\left(2 \xi_{t x}-\tau_{t t}\right)=0, \quad G\left(-t \xi_{x}+2 t \tau_{t}-\tau\right)-t^{2} \xi_{t t}=0 .
\end{gathered}
$$

Из первого уравнения найдем $\tau=e^{-D x / t} k(t)+m(t)$, подставим во второе и получим

$$
\xi=e^{D x / t} n(t)+l(t)+x \frac{m(t)}{t}+\left(-\frac{k(t) x}{t}+\frac{2 k(t)}{D}\right) e^{-D x / t} .
$$

Осталось учесть третье и четвертое уравнения. Подставим полученные выражения в третье уравнение, получим

$$
\begin{aligned}
& \frac{2 D n}{t} e^{D x / t}+\frac{2 D x}{t}\left(m_{t}-\frac{m}{t}\right)+2 D l_{t}-2 m_{t}+\frac{2 m}{t}+t m_{t t}+ \\
& \quad+\left(-\frac{3 D^{2} x^{2} k}{t^{3}}+x\left(\frac{14 D k}{t^{2}}-\frac{2 D k_{t}}{t}\right)-\frac{3(G D+2) k}{t}+10 k_{t}+t k_{t t}\right) e^{-D x / t}=0 .
\end{aligned}
$$

Из этого соотношения следует (так как $D \neq 0$ ), что $k=n=0, m_{t}-m / t=0$, $2 D l_{t}-2 m_{t}+2 m / t+t m_{t t}=0$. Таким образом, $m=m_{1} t, l=l_{1}$ и алгебра $\tau=m_{1} t$, $\xi=m_{1} x+l_{1}$ двумерна.

5. Если $Q=A=B=D=0, E \neq 0$, то $\tau=k(t) x+m(t)$. С помощью замены вида 1 получаем $T(c)=E c$. Функцию такого вида можно свести к нулевой заменой вида 2.

6. Если $Q=A=B=D=E=0$, то $T(c)=G$. Уравнение с постоянной функцией $T(c)$ может быть сведено к уравнению с нулевой функцией заменой вида 3 (с $M=0, m=1 / G)$.

Таким образом, мы получаем, что в коммутативном случае учетны все функции вида $\mathcal{F}=\left(Q c^{5}+A c^{4}+B c^{3}+D c^{2}+E c+G\right) / t$ с ненулевой алгеброй симметрий, кроме $\mathcal{F}=\left(B c^{3}-c / 2\right) / t$ с алгеброй $\tau=m_{1} x t+m_{2} t, \xi=m_{1} x^{2} / 2+m_{2} x+l_{2}$. Попробуем с помощью допустимых замен переменной упростить вид функции. Заменами вида 2 и 4 приведем ее к виду $\mathcal{F}(x)=A / x^{3}$, соответствующая алгебра симметрий будет иметь вид $\tau=m_{1} t^{2} / 2+m_{2} t+l_{2}, \xi=\left(m_{1} t+m_{2}\right) x / 2$. Лемма доказана.

ЛЕмма 8. Для функиий вида $T(c)=A c^{4}+B c^{3}+D c^{2}+E c+G+K /(c+M) c K \neq 0$, не приводимых заменой переменных $к$ уже рассмотренным, алгебра симметрий двумерна.

ДокАЗАтЕЛьство. Аналогично коммутативному случаю, используя замену вида 1 , можно считать, что $M=0$. Подставим функцию $T(c)$ заданного вида в $(27)$ 
и приведем к общему знаменателю, получим

$$
\begin{aligned}
& -c^{2} t\left(4 A c^{3}+3 B c^{2}+2 D c+E\right)\left(\xi_{t}+c \xi_{x}-c \tau_{t}-c^{2} \tau_{x}\right)+K t\left(\xi_{t}+c \xi_{x}-c \tau_{t}-c^{2} \tau_{x}\right)+ \\
& \quad+c^{2}\left(A c^{4}+B c^{3}+D c^{2}+E c+G\right)\left(t \xi_{x}-2 t \tau_{t}-3 t c \tau_{x}+\tau\right)+ \\
& \quad+K c\left(t \xi_{x}-2 t \tau_{t}-3 t c \tau_{x}+\tau\right)+t^{2} c^{2}\left(\xi_{t t}+c\left(2 \xi_{t x}-\tau_{t t}\right)+c^{2}\left(\xi_{x x}-2 \tau_{t x}\right)-c^{3} \tau_{x x}\right)=0 .
\end{aligned}
$$

В левой части уравнения стоит многочлен по $c$, тождественно равный нулю. Приравняем к нулю коэффициенты при $c^{0}$ и $c^{1}$, получим $K \xi_{t}=0, K\left(2 t \xi_{x}-3 t \tau_{t}+\tau\right)=0$. Так как $K \neq 0$, поделим второе соотношение на $t$ и продифференцируем по $t$, получим $3 t^{2} \tau_{t t}-t \tau_{t}+\tau=0$, т. е. $\tau=t f(x)+t^{1 / 3} g(x), \xi(x)=\int f(x) d x$. Подставим эти формулы в (32) и снова получим многочлен по переменной $c$. Выпишем коэффициенты при степенях $c$ :

$$
\begin{gathered}
A g=0, \quad A\left(t f_{x}+t^{1 / 3} g_{x}\right)=0, \quad 4 B g-3 D\left(t^{5 / 3} f_{x}+t g_{x}\right)-3\left(t^{8 / 3} f_{x x}+t^{2} g_{x x}\right)=0, \\
3 D g=3 t^{5 / 3}(2 E+1) f_{x}+2 t(3 E+1) g_{x}, \\
2(3 E+1) g=27 G\left(t^{5 / 3} f_{x}+t g_{x}\right), \quad G g=12 K\left(t^{5 / 3} f_{x}+t g_{x}\right) .
\end{gathered}
$$

Пусть $A \neq 0$, тогда $g=f_{x}=0$ и алгебра двумерна. Пусть $A=0$. В оставшихся четырех равенствах явно выделена зависимость от переменной $t$. Из последнего уравнения (так как $K \neq 0$ ) следует, что $G g=f_{x}=g_{x}=0$. При $g=0$ алгебра двумерна, поэтому считаем $G=0$. Аналогично из остальных уравнений получаем $E=-1 / 3, D=0$ и $B=0$, т. е. для функции $T(c)=-c / 3+K / c$ алгебра симметрий $\tau=f t+g t^{1 / 3}, \xi=f x+C$ трехмерна. Воспользуемся заменой переменных вида 2 и приведем уравнение с $T(c)=-c / 3+K / c$ к уравнению с $\mathcal{F}(c)=K / c$, а далее с помощью замены переменных вида $4-$ к уравнению с $\mathcal{F}(c)=K c^{4}$, алгебра симметрий которого найдена в лемме 1. Лемма доказана.

Лемма 9. Для функиий вида $T(c)=A\left(c^{2}+g\right)^{3 / 2}+c\left(c^{2}+g\right) / g c A \neq 0, g \neq 0$, алгебра симметрий трехмерна и ее базис есть $\Xi_{1}=\partial_{x}, \Xi_{2}=t \partial_{t}+x \partial_{x}, \Xi_{3}=$ $t x \partial_{t}+\left(x^{2}-g t^{2}\right) / 2 \partial_{x}$.

ДокАЗАтЕЛЬСтво. Заметим, что $3 c T=T_{c}\left(c^{2}+g\right)-\left(c^{2}+g\right)$. Подставим функцию $T$, выраженную через $T_{c}$, в $(27)$ :

$$
\begin{aligned}
& T_{c}\left(-3 t\left(c \xi_{t}+c^{2}\left(\xi_{x}-\tau_{t}\right)-c^{3} \tau_{x}\right)+\left(c^{2}+g\right)\left(t \xi_{x}-2 t \tau_{t}-3 t c \tau_{x}+\tau\right)\right)- \\
& \quad-\left(c^{2}+g\right)\left(t \xi_{x}-2 t \tau_{t}-3 t c \tau_{x}+\tau\right)+3 t^{2}\left(c \xi_{t t}+c^{2}\left(2 \xi_{t x}-\tau_{t t}\right)+c^{3}\left(\xi_{x x}-2 \tau_{t x}\right)-c^{4} \tau_{x x}\right)=0 .
\end{aligned}
$$

Поскольку $T_{c}$ не является дробно-рациональной функцией, в силу линейной независимости $T_{c}$ и дробно-рациональной функции коэффициент при $T_{c}$ равен нулю, следовательно, второе слагаемое тоже равно нулю, и мы получаем

$$
\begin{gathered}
t \xi_{x}-2 t \tau_{t}+\tau=0, \quad \xi_{t}+g \tau_{x}=0, \quad 2 t \xi_{x}-t \tau_{t}-\tau=0, \quad g \tau_{x}+t \xi_{t t}=0, \\
t \xi_{x}-2 t \tau_{t}+\tau-3 t^{2}\left(2 \xi_{t x}-\tau_{t t}\right)=0, \quad \tau_{x}+t\left(\xi_{x x}-2 \tau_{t x}\right)=0, \quad \tau_{x x}=0 .
\end{gathered}
$$

Из первого, третьего и седьмого уравнений системы следует, что $\tau=k_{1} x t+k_{2} t$. Из второго уравнения мы имеем $\xi=-g k_{1} t^{2} / 2+l(x)$. Подставим это соотношение 
в первое уравнение, получим $l=k_{1} x^{2} / 2+k_{2} x+l_{1}$. Таким образом, функции $T(c)$ заданного в условии леммы вида соответствует трехмерная алгебра симметрий

$$
\tau=k_{1} x t+k_{2} t, \quad \xi=\frac{k_{1}\left(x^{2}-g t^{2}\right)}{2}+k_{2} x+l_{1} .
$$

Последовательно применяя замены переменных типа 2, 4, 5, можно привести уравнение с указанной в условии леммы функцией $T(c)$ к уравнению с функцией $\mathcal{F}=A\left(1+\frac{(t+g c)^{2}}{t^{2}+2 g x}\right)^{3 / 2} ;$ соответствующая алгебра будет иметь вид

$$
\tau=-g k_{1} x+k_{2} t+l_{1}, \quad \xi=k_{1}\left(3 t x+\frac{t^{3}}{g}\right)+2 k_{2} x-\frac{l_{1} t}{g} .
$$

Лемма доказана.

ЛЕмма 10. Для функиий вида $T(c)=A c^{a}+p c$ c $A \neq 0, a \neq-1,0, \ldots, 5$, не приводимых заменой переменных к уже рассмотренным, алгебра симметрий двумерна.

ДокАЗАТЕЛЬСТво. Подставим функцию $T(c)$ заданного вида в (27), получим

$$
\begin{aligned}
-a A c^{a-1} t \xi_{t} & +A c^{a}\left((a-2) t \tau_{t}-(a-1) t \xi_{x}+\tau\right)+(a-3) A c^{a+1} t \tau_{x}+\left(t^{2} \xi_{t t}-p t \xi_{t}\right)+ \\
& +c\left(t^{2}\left(2 \xi_{t x}-\tau_{t t}\right)-p t \tau_{t}+p \tau\right)+c^{2}\left(t^{2}\left(\xi_{x x}-2 \tau_{t x}\right)-2 p t \tau_{x}\right)-c^{3} t^{2} \tau_{x x}=0 .
\end{aligned}
$$

Так как случаи $a=-1,0,1, \ldots, 5$ уже рассмотрены в леммах 7 и 8 , степени при $c$ различны, и мы получаем систему

$\xi_{t}=0, \quad \tau_{x}=0, \quad(a-2) t \tau_{t}-(a-1) t \xi_{x}+\tau=0, \quad t^{2} \tau_{t t}+p\left(t \tau_{t}-\tau\right)=0, \quad \xi_{x x}=0$.

Если $p \neq-1$, то из последних двух уравнений найдем, что $\tau=k t+m t^{-p}, \xi=n x+l$, подставим в третье уравнение, получим $(a-1)(k-n) t+(1-(a-2) p) m t^{-p}=0$. Тогда $k=n,(1-(a-2) p) m=0$. При этом либо $m=0$ и алгебра двумерна, либо $p=1 /(a-2)$ и для $T(c)=A c^{a}+c /(a-2)$ алгебра симметрий $\tau=k t+m t^{-1 /(a-2)}$, $\xi=k x+l$ трехмерна. Воспользуемся заменой переменных вида 2 , которая сводит $T(c)=A c^{a}+c /(a-2)$ к коммутативному случаю $\mathcal{F}(c)=A c^{a}$.

Если $p=-1$, то из последних двух уравнений найдем, что $\tau=k t+m t \ln t$, $\xi=n x+l$, и подставим эти равенства в третье уравнение, в результате получим $((a-1) k+(a-2) m-(a-1) n) t+(a-1) m t \ln t=0$. Отсюда $m=0, k=n$ и алгебра двумерна. Лемма доказана.

ЛЕмма 11. Для функиий вида $\mathcal{F}(c)=A e^{a c}+p, A \neq 0, a \neq 0$, не приводимых заменой переменных к уже рассмотренным, алгебра симметрий двумерна.

ДокАзАТЕЛьСтво. Подставим функцию $T(c)$ заданного вида в (27), получим

$$
\begin{aligned}
A e^{a c}\left(\left(t \xi_{x}\right.\right. & \left.\left.-2 t \tau_{t}+\tau-a t \xi_{t}\right)-c\left(a t \xi_{x}-a t \tau_{t}+3 t \tau_{x}\right)+a c^{2} t \tau_{x}\right)+p t \xi_{x}-2 p t \tau_{t}+ \\
& +p \tau+t^{2} \xi_{t t}+c\left(2 t^{2} \xi_{t x}-t^{2} \tau_{t t}-3 p t \tau_{x}\right)+c^{2}\left(t^{2} \xi_{x x}-2 t^{2} \tau_{t x}\right)-c^{3} t^{2} \tau_{x x}=0 .
\end{aligned}
$$

Поскольку $T$ не является дробно-рациональной функцией, в силу линейной независимости $T$ и дробно-рациональной функции коэффициент при $T$ и оставшееся слагаемое равы нулю, тогда

$$
\begin{gathered}
t \xi_{x}-2 t \tau_{t}+\tau-a t \xi_{t}=0, \quad a t \xi_{x}-a t \tau_{t}+3 t \tau_{x}=0, \quad \tau_{x}=0, \\
p t \xi_{x}-2 p t \tau_{t}+p \tau+t^{2} \xi_{t t}=0, \quad 2 \xi_{t x}-\tau_{t t}=0, \quad \xi_{x x}=0
\end{gathered}
$$


Отсюда находим $\tau=k t+m, \xi=k x+l(t)$ и $l_{t}=m / a t, p m+t^{2} l_{t t}=0$. Тогда $l=(m \ln t) / a+l_{1}$ и $m(p-1 / a)=0$. Если $m=0$, то алгебра двумерная. Если $m \neq 0$, то $p=1 / a$, и тогда $\tau=k t+m, \xi=k x+m \ln t / a+l_{1}$ есть трехмерная алгебра для $T(c)=A e^{a c}+1 / a$. Заменой вида 3 функция $T(c)=A e^{a c}+1 / a$ приводится к коммутативному случаю $\mathcal{F}(c)=A e^{a c}$. Лемма доказана.

\section{8. Доказательство точной двумерности алгебры симметрий в осталь-} ных случаях. Докажем, что все функции $T(c)$ с алгеброй симметрий размерности более двух исчерпываются разобранными в леммах 7-11. Будем рассматривать (27) как уравнение относительно $T$ вида $-T_{c} A+T B+D=0$. Возможны следующие варианты.

1. Пусть $A=0$. Тогда $\tau_{x}=\xi_{t}=0$, a $\tau_{t}=\xi_{x}=$ const, $\tau=C_{1} t+C_{3}$ и $\xi=$ $C_{1} x+C_{2}$. Постоянная $C_{1}$ отлична от нуля в силу предположения, что хотя бы одна из функций $\tau, \xi$ не является постоянной. Но тогда оказывается, что $B=C_{3}, D=0$, а значит, $T=0$, что уже рассмотрено в лемме 7 .

2. Пусть $A \neq 0$. Дифференцируя соотношение $-T_{c} A+T B+D=0$ по $x$ и по $t$, получим систему из трех уравнений относительно $T(c)$ и $T_{c}(c)$ :

$$
-T_{c} A+T B+D=0, \quad-T_{c} A_{x}+T B_{x}+D_{x}=0, \quad-T_{c} A_{t}+T B_{t}+D_{t}=0 .
$$

Возможны три случая: система (33) несовместна, все три уравнения эквивалентны или среди уравнений есть хотя бы два непропорциональных друг другу. Первый случай нас интересовать далее не будет, так как функций $T(c)$, удовлетворяющих этой системе, не существует. Рассмотрим оставшиеся два случая.

2.1. Пусть $B A_{x}=B_{x} A$ и $B A_{t}=B_{t} A$, тогда $B$ пропорциональна $A$ с коэффициентом, зависящим только от $c: \phi(c) t\left(\xi_{t}+c \xi_{x}-c \tau_{t}-c^{2} \tau_{x}\right)=t\left(\xi_{x}-2 \tau_{t}-3 c \tau_{x}\right)+\tau$.

2.1.1. Пусть $\tau_{x} \not \equiv 0$, тогда, фиксируя соответствующие значения $(t, x)$, получаем, что $\phi(c)=(3 c+a) /\left(c^{2}+b c+d\right)$, где $a, b, d-$ некоторые константы. Используем полученный анзац в (27), получим

$$
\begin{aligned}
& t(3 c+a)\left(\xi_{t}+c \xi_{x}-c \tau_{t}-c^{2} \tau_{x}\right)=\left(c^{2}+b c+d\right)\left(\left(t \xi_{x}-2 t \tau_{t}+\tau\right)-3 t c \tau_{x}\right), \\
& t\left(a \xi_{t}+c\left(3 \xi_{t}+a \xi_{x}-a \tau_{t}\right)+c^{2}\left(3 \xi_{x}-3 \tau_{t}-a \tau_{x}\right)\right)= \\
& \quad=c^{2}\left(t \xi_{x}-2 t \tau_{t}+\tau\right)+b c\left(t \xi_{x}-2 t \tau_{t}+\tau\right)+d\left(t \xi_{x}-2 t \tau_{t}+\tau\right)-3 b c^{2} t \tau_{x}-3 d c t \tau_{x} .
\end{aligned}
$$

Коэффициенты при степенях $c$ приравняем к нулю, в результате имеем

$$
\begin{gathered}
2 t \xi_{x}-\tau=t(a-3 b) \tau_{x}+t \tau_{t}, \quad 3 t \xi_{t}+t(a-b) \xi_{x}-b \tau=t(a-2 b) \tau_{t}-3 d t \tau_{x}, \\
a t \xi_{t}=d t \xi_{x}-2 d t \tau_{t}+d \tau .
\end{gathered}
$$

Сделаем замену переменных $\bar{t}=t, \bar{x}=x-(a-3 b) t / 3, \bar{c}=c-(a-3 b) / 3$. При такой замене получим новые функции $\bar{\tau}(\bar{t}, \bar{x})=\tau(t, x), \bar{\xi}(\bar{t}, \bar{x})=\xi-(a-3 b) \tau / 3$, соотношение (27) перейдет в себя, а полученные уравнения примут вид

$$
\begin{gathered}
\xi_{x}=\frac{\tau}{2 t}+\frac{\tau_{t}}{2}, \quad \xi_{t}=-\left(\frac{a(a-3 b)}{9}+d\right) \tau_{x} \\
\left(\frac{a(a-3 b)}{9}+d\right)\left(2\left(b-\frac{2 a}{3}\right) t \tau_{x}+t \tau_{t}-\tau\right)=0 .
\end{gathered}
$$


2.1.1(a). Предположим сначала, что $9 d+a(a-3 b)=0$, и тогда $\xi_{x}=\tau / 2 t+\tau_{t} / 2$, $\xi_{t}=0$. Выпишем условие совместности: $\tau_{t t}+\tau_{t} / t-\tau / t^{2}=0$. Найдем из него $\tau=k(x) t+m(x) / t$, при этом формулы для производных функции $\xi$ упрощаются: мы имеем $\xi_{t}=0, \xi_{x}=k(x)$, т. е. $\xi=\int k(x) d x$. Подставим эти выражения в $(27)$ :

$$
\left(m-c t^{3} k_{x}-c t m_{x}\right)\left(3 T-T_{c} c\right)-c^{2} t^{3} k_{x}-c^{3} t^{4} k_{x x}-2 c m+2 c^{2} t m_{x}-c^{3} t^{2} m_{x x}=0 .
$$

Мы получили в левой части равенства многочлен по $t$, поэтому

$k_{x x}=0, \quad k_{x}\left(3 T-T_{c} c+c\right)=0, \quad m_{x x}=0, \quad m_{x}\left(3 T-T_{c} c-2 c\right)=0, \quad m\left(3 T-T_{c} c-2 c\right)=0$, т. е. $k=k_{1} x+k_{2}, m=m_{1} x+m_{2}$. Пусть $3 T-T_{c} c-2 c=0$, тогда $T=M c^{3}+c$. Если $3 T-T_{c} c-2 c \neq 0$, то $m=0$ и либо $T(c)=M c^{3}-c / 2$, либо $k=k_{2}$; в результате мы получаем двумерную алгебру. Алгебры для таких функций $T(c)$ найдены в лемме 7 .

2.1.1б. Предположим, что $9 d+a(a-3 b) \neq 0$, тогда $2(b-2 a / 3) t \tau_{x}+t \tau_{t}-\tau=0$. Найдем $\tau=t k(x-2(b-2 a / 3) t)$. Подставим это выражение в формулы для производных функции $\xi$; имеем

$$
\xi_{x}=k-\left(b-\frac{2 a}{3}\right) t k^{\prime}, \quad \xi_{t}=-\left(\frac{a(a-3 b)}{9}+d\right) t k^{\prime} .
$$

Условие совместности записывается как $9(3 b-2 a) k^{\prime}=\left(2(3 b-2 a)^{2}+a(a-3 b)+9 d\right) t k^{\prime \prime}$, откуда получаем $\left(2(3 b-2 a)^{2}+a(a-3 b)+9 d\right) k^{\prime \prime}=(2 a-3 b) k^{\prime}=0$. В силу сделанных выше предположений возможны только два случая.

Либо $k^{\prime}=0$, т. е. $k=$ const, при этом $\xi_{x}=k, \xi_{t}=0$, тем самым $\tau=k t, \xi=k x+C$ есть двумерная алгебра.

Либо $k^{\prime} \neq 0$, тогда $k^{\prime \prime}=0$ и $a=3 b / 2$, т. е. $k=k_{1} x+k_{2}$. При этом $\xi_{x}=k_{1} x+k_{2}$, $\xi_{t}=-g k_{1} t$, где $g=d-b^{2} / 4$. Отсюда $\tau=k_{1} t x+k_{2} t, \xi=k_{1}\left(x^{2}-g t^{2}\right) / 2+k_{2} x+m$. Подставим эти выражения в (27), получим $k_{1}\left[T_{c}\left(g+c^{2}\right)-3 T c-\left(g+c^{2}\right) k_{1}\right]=0$. Если $k_{1}=0$, то алгебра двумерная. Предполагаем далее $k_{1} \neq 0$, тогда

$$
T(c)=\exp \left(\int \frac{3 c d c}{c^{2}+g}\right)\left(M+\int e^{-\int \frac{3 c d c}{c^{2}+g}} d c\right)=M\left(c^{2}+g\right)^{3 / 2}+\frac{c\left(c^{2}+g\right)}{g}
$$

(если $g=0$, то получаем функцию $T=M c^{3}-c / 2$ с уже изученной алгеброй симметрий). Функция $T(c)$ вида (34) рассмотрена в лемме 9.

2.1.2. Пусть $\tau_{x} \equiv 0, \xi_{t} \not \equiv 0$. Тогда $\phi(c)=a /(1+b c)$ и выполнено соотношение

$$
a t\left(\xi_{t}+c \xi_{x}-c \tau_{t}\right)=\left(t\left(\xi_{x}-2 \tau_{t}\right)+\tau\right)(1+b c) .
$$

Приравняем к нулю коэффициенты при степенях $c$ в получившемся многочлене, получим $a t \xi_{t}=t \xi_{x}-2 t \tau_{t}+\tau,(b-a) t \xi_{x}+(a-2 b) t \tau_{t}+b \tau=0$. Выразим из первого уравнения $\tau_{t}$ и подставим во второе, имеем

$$
\tau_{t}=\frac{1}{2} \xi_{x}-\frac{a}{2} \xi_{t}+\frac{1}{2 t} \tau, \quad a\left(t \xi_{x}+t(a-2 b) \xi_{t}-\tau\right)=0 .
$$

2.1.2а. В случае $a=0$ получаем $\tau=\tau(t), \xi=\left(2 \tau_{t}-\tau / t\right) x+C(t)$. Подставим эти соотношения в $(27)$, получим

$$
\begin{aligned}
& T_{c}\left(\left(2 t \tau_{t t}-\tau_{t}+\frac{\tau}{t}\right) x+t C_{t}+c t \tau_{t}-c \tau\right)- \\
& \quad-\left(2 t^{2} \tau_{t t t}-t \tau_{t t}+2 \tau_{t}-\frac{2 \tau}{t}\right) x+t^{2} C_{t t}+c\left(3 t^{2} \tau_{t t}-2 t \tau_{t}+2 \tau\right)=0 .
\end{aligned}
$$


В левой части равенства мы имеем многочлен по $x$, т. е.

$$
\begin{gathered}
T_{c}\left(2 t \tau_{t t}-\tau_{t}+\frac{\tau}{t}\right)-\left(2 t^{2} \tau_{t t t}-t \tau_{t t}+2 \tau_{t}-\frac{2 \tau}{t}\right)=0 . \\
T_{c}\left(t C_{t}+c\left(t \tau_{t}-\tau\right)\right)-t^{2} C_{t t}-c\left(3 t^{2} \tau_{t t}-2 t \tau_{t}+2 \tau\right)=0 .
\end{gathered}
$$

Либо из первого уравнения находим $T(c)=E c+G$, что уже рассмотрено выше, либо коэффициент при $T$ равен нулю, $2 t^{2} \tau_{t t}-t \tau_{t}+\tau=0$, и тогда $\tau=k t^{1 / 2}+m t$. В этом случае рассмотрим второе уравнение: $2 T_{c}\left(2 t^{1 / 2} C_{t}-c k\right)=4 t^{3 / 2} C_{t t}+c k$. Из условия разрешимости уравнения либо $k=0$ и снова $T=E c+G$, либо $C_{t}+2 t C_{t t}=0$ и $T=-c / 2+G$, что также уже рассмотрено выше.

2.1.2б. В случае $a \neq 0$ при $a=2 b$ получается $\xi=x \tau / t+C(t)$. Из первого уравнения в $(35)$ находим $2 t^{2} \tau_{t}=2 t \tau-a x\left(t \tau_{t}-\tau\right)-a t^{2} C_{t}$. Пользуясь тем, что $\tau_{x}=0$, получаем $t \tau_{t}-\tau=0$, т. е. $\tau=k t, C=$ const, при этом алгебра двумерна для любой функции $T$. При $a \neq 2 b$ мы имеем

$$
\xi=\int \frac{\tau d t}{t(a-2 b)}+C(t+(2 b-a) x), \quad \tau_{t}+\frac{b}{(a-2 b) t} \tau=(b-a) C^{\prime} .
$$

Если при этом $a=b$, то $\tau=k t$ и $\xi=-k t / a+C(t+a x)$, и из соотношения $(27)$

$$
-T_{c}\left(a C^{\prime}-k\right)(1+a c)+a T\left(a C^{\prime}-k\right)+a t(1+a c)^{2} C^{\prime \prime}=0
$$

следует, что $T(c)=(1+a c)(Q c+M)$. Этот случай уже рассмотрен выше.

Если $a \neq b$, то, пользуясь тем, что $\tau=\tau(t)$, находим $C=C_{1}(t+(2 b-a) x)+C_{2}$ и $\tau=k t^{b /(2 b-a)}+C_{1} t$. Также (при $\left.b \neq 0\right)$ мы имеем $\xi=-k t^{b /(2 b-a)} / b+C_{1} x+C_{2}$. Подставим эти соотношения в (27), получим

$$
k\left(T_{c}(1+c b)-a T-\frac{(a-b)(1+c b)}{2 b-a}\right)=0 .
$$

Мы считаем, что $k \neq 0$, иначе алгебра двумерна, и находим

$$
T(c)=M(1+c b)^{a / b}-\frac{1+c b}{2 b-a} .
$$

С точностью до замены переменных из группы эквивалентности получаем функцию вида $T=M c^{p}+c /(p-2)$, ее алгебра симметрий найдена в лемме 10 .

Если $b=0$, то $\tau=k-C_{1} a t$ и $\xi=k \ln t / a-C_{1} a x+C_{2}$. Подставим эти соотношения в $(27)$, имеем $T_{c}-T a+1=0$, откуда получаем функцию $T(c)=M e^{a c}+1 / a$, которая рассмотрена в лемме 11 и имеет трехмерную алгебру симметрий.

2.1.3. Пусть $\tau_{x} \equiv 0, \xi_{t} \equiv 0$. В этом случае в силу предположения $A \not \equiv 0$ выполнено условие $\xi_{x}-\tau_{t} \not \equiv 0$, и мы имеем $\phi(c)=a / c$; тогда $(a-1) \xi_{x}=(a-2) \tau_{t}+\tau / t=$ const $=l$. Если $a=2$, то $\tau=l t, \xi=l x+m$, и это противоречит предположению $\xi_{x}-\tau_{t} \not \equiv 0$. Если $a=1$, то $\tau=b t, \xi=\xi(x)$. Подставим эти соотношения в $(27)$, получим $\left(T-T_{c} c\right)\left(\xi_{x}-b\right)+t c^{2} \xi_{x x}=0$, откуда $\xi=l x+m$ и либо $l=b$ (алгебра двумерная), либо $T(c)=M c$. Алгебра симметрий для этой функции найдена в лемме 7 .

Если $a \neq 1$ и $a \neq 2$, то $\tau=b t^{-1 /(a-2)}+l t /(a-1), \xi=l x /(a-1)+m$. Вернемся к соотношению (27), которое теперь приобретает вид

$$
b\left(T_{c} c-T a+c \frac{a-1}{a-2}\right)=0 .
$$


Случай $b=0$ приводит к двумерной алгебре. Иначе, считая, что $b \neq 0$, получаем $T(c)=M c^{a}+c /(a-2) ;$ соответствующая алгебра симметрий найдена в лемме 10.

2.2. Пусть среди уравнений системы (33) есть хотя бы два непропорциональных друг другу (одно из соотношений $B A_{x}=B_{x} A$ и $B A_{t}=B_{t} A$ не выполнено тождественно). Тогда мы сможем найти анзац $T(c)$ : так как $A \neq 0$, из соответствующей пары уравнений при некоторых фиксированных значениях $(t, x)$ мы получаем, что $T$ - это частное двух многочленов (в числителе стоит многочлен пятой степени, в знаменателе - второй). И, так как $B \neq 0$, из той же пары уравнений при соответствующих значениях $(t, x)$ получаем, что $T_{c}$ - это частное двух многочленов (в числителе стоит многочлен четвертой степени, в знаменателе - второй). Дифференцируя выражение для $T$ по $c$ и сравнивая с видом $T_{c}$, получаем, что возможны только два случая: в знаменателе обеих функций стоит константа или после выделения целой части дробь сокращается. То есть возможны следующие функции $T$ :

$$
T(c)=Q c^{5}+A c^{4}+B c^{3}+D c^{2}+E c+G, \quad T(c)=A c^{4}+B c^{3}+D c^{2}+E c+G+\frac{K}{c} .
$$

Функции такого вида уже рассмотрены в леммах 7 и 8.

Таким образом, мы показали, что все случаи, когда имеется более чем двумерная алгебра симметрий, рассмотрены в леммах предыдущего пункта.

4.9. Уравнения с одномерными алгебрами симметрий. Если алгебра симметрий одномерна, то заменой переменных порождающий оператор можно привести к виду $\Xi=\partial_{x}$. Алгебре такого вида соответствует уравнение $(1)$ с $\mathcal{F}(t, c)$. Поэтому семейство функций $\mathcal{F}$, для которых алгебра симметрий уравнения одномерна, состоит с точностью до преобразований из группы эквивалентности из функций $\mathcal{F}(t, c)$, не сводящихся к функциям из семейств, приведенных в табл. 1-3. Отметим, что замена $\bar{t}=x, \bar{x}=t$, меняющая ролями "пространство" и "время", позволяет нам вместо $\mathcal{F}(t, c)$ рассматривать $\mathcal{F}(x, c)$ с одномерной алгеброй с базисом $\Xi=\partial_{t}$.

Теорема 3 полностью доказана.

\section{Список литературы}

[1] I. Müller, T. Ruggeri, Extended Thermodynamics, Springer Tracts in Natural Philosophy, 37, Springer, New York, 1998.

[2] Л. В. Овсянников, "Групповые свойства уравнения Чаплыгина", Прикладная механика и техническая физика, 1:3 (1960), 126-145.

[3] K.С. Платонова, "Групповой анализ уравнения Больцмана. I. Группы симметрий", Дифферени. уравнения, 53:4 (2017), 538-546.

[4] Ю.Н. Григорьев, С. В. Мелешко, "Полная группа Ли и инвариантные решения системы уравнений Больцмана многокомпонентной смеси газов", Сиб. матем. журн., 38:3 (1997), 510-525.

[5] К. С. Платонова, "Групповой анализ уравнения Больцмана. II. Группы эквивалентности и группы симметрий в специальном случае", Дифферени. уравнения, 53:6 (2017), 801-813.

[6] С. Ли, Симметрии дифференииальных уравнений, т. 2: Лекции о непрерывных группах с геометрическими и другими приложениями, РХД, М.-Ижевск, 2011.

[7] A. González-López, N. Kamran, P. J. Olver, "Lie algebras of vector fields in the real plane", Proc. London Math. Soc., s3-64:2 (1992), 339-368. 